\title{
On the algebraic classification of module spectra
}

\author{
IRAKLI PATCHKORIA
}

\begin{abstract}
Using methods developed by Franke in [7], we obtain algebraic classification results for modules over certain symmetric ring spectra ( $S$-algebras). In particular, for any symmetric ring spectrum $R$ whose graded homotopy ring $\pi_{*} R$ has graded global homological dimension 2 and is concentrated in degrees divisible by some natural number $N \geq 4$, we prove that the homotopy category of $R$-modules is equivalent to the derived category of the homotopy ring $\pi_{*} R$. This improves the Bousfield-Wolbert algebraic classification of isomorphism classes of objects of the homotopy category of $R$-modules. The main examples of ring spectra to which our result applies are the $p$-local real connective $K$-theory spectrum $k o_{(p)}$, the Johnson-Wilson spectrum $E(2)$, and the truncated Brown-Peterson spectrum $B P\langle 1\rangle$, all for an odd prime $p$. We also show that the equivalences for all these examples are exotic in the sense that they do not come from a zigzag of Quillen equivalences.
\end{abstract}

18E30, 55P42, 55P43; 18G55

\section{Introduction}

In [2] Bousfield gave an algebraic classification of isomorphism classes of objects in the $E(1)$-local (or, equivalently, $K_{(p)}$-local) stable homotopy category $L_{1} \mathbf{S}$ for an odd prime $p$. For this he used a certain $k$-invariant coming from a $d_{2}$-differential of the $E(1)-$ homology Adams spectral sequence. Bousfield also tried to describe morphisms in $L_{1} \mathbf{S}$ but only managed to characterize them up to Adams filtration.

In [7] Franke, using the language of triangulated derivators, proved an abstract uniqueness theorem for triangulated categories possessing an Adams spectral sequence. Applying this result to $L_{1} \mathbf{S}$ (for an odd prime), he obtained an equivalence of categories between $L_{1} \mathbf{S}$ and the derived category of certain twisted chain complexes of $E(1)_{*} E(1)$-comodules. (For a streamlined exposition of this result see Roitzheim [19].) This gives a complete algebraic description of the category $L_{1} \mathbf{S}$ and thus improves the aforementioned result of Bousfield. Moreover, as is shown in [19], this equivalence is exotic in the sense that it does not come from a zigzag of Quillen equivalences.

A similar classification result to that of Bousfield for module spectra was obtained by Wolbert in [25]. More precisely, let $R$ be a symmetric ring spectrum (or an $S$ algebra) and suppose the (right) graded global homological dimension of the homotopy 
ring $\pi_{*} R$ is less or equal than two. Suppose further that $\pi_{*} R$ is concentrated in dimensions divisible by some natural number $N \geq 2$. Under these assumptions, Wolbert gives an algebraic characterization of isomorphism classes of objects in the derived category $\mathcal{D}(R)$ [25, Theorem 6]. The main example of a ring spectrum to which this can be applied is the $p$-local real connective $K$-theory spectrum $k o_{(p)}$ for an odd prime $p$.

Note that Wolbert, like Bousfield, did not give a complete algebraic characterization of morphisms in $\mathcal{D}(R)$. Consequently, a natural question arises whether one can apply Franke's methods to improve Wolbert's classification. One of the aims of the present paper is to examine this question.

Let $R$ be a symmetric ring spectrum. We say that an object $X$ of $\mathcal{D}(R)$ has dimension $k$ if the projective dimension of the homotopy $\pi_{*} X$ regarded as a graded $\pi_{*} R$-module is equal to $k$. Similarly, we say that an object $M$ in the derived category $\mathcal{D}\left(\pi_{*} R\right)$ (ie, a differential graded $\pi_{*} R$-module) has dimension $k$ if the projective dimension of the homology $H_{*} M$ regarded as a graded $\pi_{*} R$-module is equal to $k$. The main aim of this paper is to give self-contained homotopy theoretic proofs of the following theorems.

Theorem 1.1.1 Let $R$ be a symmetric ring spectrum. Suppose that the graded homotopy ring $\pi_{*} R$ is concentrated in dimensions divisible by a natural number $N$ and assume that the (right) graded global homological dimension of $\pi_{*} R$ is less than $N$. Then one can construct a functor

$$
\mathscr{R}: \mathcal{D}\left(\pi_{*} R\right) \longrightarrow \mathcal{D}(R)
$$

such that the following hold:

(i) The diagram

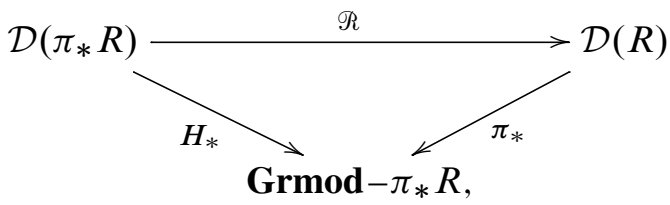

where Grmod $-\pi_{*} R$ denotes the category of (right) graded $\pi_{*} R-$ modules, commutes up to a natural isomorphism.

(ii) The functor $\mathscr{R}: \mathcal{D}\left(\pi_{*} R\right) \longrightarrow \mathcal{D}(R)$ restricts to an equivalence of the full subcategories of at most one-dimensional objects.

(iii) Let $X \in \mathcal{D}(R)$ and suppose that $X$ has dimension at most two. Then there exists $M$ in $\mathcal{D}\left(\pi_{*} R\right)$ such that $\mathscr{R}(M)$ is isomorphic to $X$ in $\mathcal{D}(R)$. 
Theorem 1.1.2 Let $R$ be a symmetric ring spectrum. Suppose that the graded homotopy ring $\pi_{*} R$ is concentrated in dimensions divisible by a natural number $N$ and assume that the (right) graded global homological dimension of $\pi_{*} R$ is less than $N-1$. Then the functor

$$
\mathscr{R}: \mathcal{D}\left(\pi_{*} R\right) \longrightarrow \mathcal{D}(R)
$$

restricts to an equivalence of the full subcategories of at most two-dimensional objects.

In particular, we have the following corollary.

Corollary 1.1.3 Let $R$ be a symmetric ring spectrum. Suppose that the graded homotopy ring $\pi_{*} R$ is concentrated in dimensions divisible by a natural number $N \geq 4$ and assume that the (right) graded global homological dimension of $\pi_{*} R$ is equal to two. Then the functor

$$
\mathscr{R}: \mathcal{D}\left(\pi_{*} R\right) \longrightarrow \mathcal{D}(R)
$$

is an equivalence of categories.

This corollary improves Wolbert's classification [25, Theorem 6] for $N \geq 4$.

Note that similar results hold for differential graded rings and our proofs work in this algebraic setting as well (see Remark 6.4.4).

The functor $\mathscr{R}: \mathcal{D}\left(\pi_{*} R\right) \longrightarrow \mathcal{D}(R)$ is in fact Franke's functor for the special case of $\mathcal{D}(R)$. Franke constructs his functor for general triangulated derivators possessing an Adams spectral sequence, and claims that it is an equivalence of categories [7, 2.2, Proposition 2]. If this is so, then under the hypotheses of Theorem 1.1.1 the functor $\mathscr{R}: \mathcal{D}\left(\pi_{*} R\right) \longrightarrow \mathcal{D}(R)$ becomes an equivalence that trivially implies Theorems 1.1.1 and 1.1.2. However, the proof of [7, 2.2, Proposition 2] contains a gap (see Remark 6.3.1) which we were unable to fill in the general setting. We managed to eliminate this gap in low dimensional cases and thus obtained the statements of Theorems 1.1.1 and 1.1.2.

Note that for the construction of $\mathscr{R}$ we adopt the methods of [19]. In particular, we use the language of model categories rather than that of derivators which is used by Franke. This technically simplifies our exposition.

For $n<2(p-1)$, the Johnson-Wilson spectra $E(n)$ are nontrivial examples of ring spectra to which Theorem 1.1.1 can be applied. Further important examples are the truncated Brown-Peterson spectra $B P\langle n\rangle$ for $n+1<2(p-1)$. (For all primes and any $n, E(n)$ as well as $B P\langle n\rangle$ admit $A_{\infty}$-structures (see eg, Lazarev [16]) and thus possess models which are symmetric ring spectra.) Note that for all these ring spectra the functor $\mathscr{R}$ is not a derived functor of a Quillen functor (see Subsection 4.2). 
Examples of ring spectra which fulfill the hypotheses of Corollary 1.1.3 are the $p$-local real connective $K$-theory spectrum $k o_{(p)}$, the Johnson-Wilson spectrum $E(2)$, and the truncated Brown-Peterson spectrum $B P\langle 1\rangle$, all for an odd prime $p$. We show in Subsection 4.2 that all these examples are exotic in the sense that the equivalence $\mathscr{R}$ does not come from a zigzag of Quillen equivalences.

The paper is organized as follows. In Section 2 some basic facts about triangulated categories and model categories are recalled. Next we briefly review stable model categories and give examples which are important for our purposes. Furthermore we recall model structures on diagram categories and long exact sequences of a homotopy pullback and homotopy coequalizer.

In Section 3 the functor $\mathscr{R}$ is constructed and Theorem 1.1.1 (i) is proved. In fact, we define $\mathscr{R}$ in a more general setting of stable model categories. The most complicated step in the construction of $\mathscr{R}$ is discussed in Subsection 3.3 which concerns Franke's crowned diagrams (see Example 2.5.5). At the end of Section 3 we verify that the functor $\mathscr{R}$ commutes with suspensions.

In Section 4 we recall the notion of a derived mapping space and prove a proposition which gives a sufficient condition for $\mathscr{R}$ not being a derived functor. We also discuss some nontrivial examples to which Theorem 1.1.1 applies.

Section 5 is devoted to the proof of Theorem 1.1.1(ii). As a corollary we get that under the hypotheses of Theorem 1.1.1 the functor $\mathscr{R}: \mathcal{D}\left(\pi_{*} R\right) \longrightarrow \mathcal{D}(R)$ is an equivalence of categories if the graded global homological dimension of $\pi_{*} R$ is equal to one. The complex $K$-theory spectrum $K U$ and the connective Morava $K$-theory spectra $k(n)$, $n \geq 1$ serve as our main examples here.

Finally, in Section 6 we prove Theorem 1.1.1(ii) and Theorem 1.1.2. We also discuss nontrivial examples to which Corollary 1.1.3 applies. Note that the key results in this section are Lemmas 6.2.1 and 6.2.4 which fill the aforementioned gap in the proof of [7, 2.2, Proposition 2] for the two dimensional case.

We conclude the paper with an appendix about infinite loop spaces where it is shown that certain infinite loop spaces are not weakly equivalent to products of EilenbergMac Lane spaces. These facts are essentially used in Subsections 4.3, 5.3 and 6.4 to deduce that for all examples, our equivalences are exotic. The material in the appendix is well known. However, as we were unable to find proper references for it, we included all the necessary proofs.

Acknowledgements This paper is based on my master's thesis at the University of Bonn. I would like to thank my advisor Stefan Schwede for suggesting this topic and 
for his help and encouragement throughout the way. Many thanks also go to Katja Hutschenreuter and Lennart Meier for their valuable comments and suggestions on earlier drafts of the paper.

This research was supported by the Deutsche Forschungsgemeinschaft Graduiertenkolleg 1150 "Homotopy and Cohomology."

\section{Preliminaries}

\subsection{Notation}

2.1.1 Simplicial objects The category of simplicial sets is denoted by sSet. For $n \geq 0, \Delta[n]$ stands for the standard simplicial $n$-simplex. Next, if $K$ is a simplicial set and $x \in K_{n}$, then we denote again by $x$ the unique simplicial map $\Delta[n] \longrightarrow K$ which sends the generator of $\Delta[n]$ to $x$.

The letter $I$ stands for $\Delta[1]$ and $I_{+}$for the union of $\Delta[1]$ with a disjoint base point. The pointed simplicial $0-$ sphere is denoted by $S^{0}$, ie, the union of the standard 0 -simplex with a disjoint base point. Further,

$$
\mathrm{in}_{0}, \mathrm{in}_{1}: S^{0} \longrightarrow I_{+}
$$

are our notation for obvious maps sending the nonbase point of $S^{0}$ to (0) and (1), respectively. $S^{1}$ denotes the simplicial circle $I /(0 \sim 1)$, ie, $\Delta[1] / \partial \Delta[1]$.

2.1.2 DG modules and graded modules For any differential graded ring $A$, we denote by Mod $-A$ the category of differential graded right $A$-modules. In particular, for any graded ring $B$, we have the category $\operatorname{Mod}-B$, where $B$ is regarded as a differential graded ring with zero differential. Further, for any graded ring $B, \operatorname{Grmod}-B$ stands for the category of graded right modules over $B$. For any $X \in \mathbf{G r m o d}-B$, proj.dim $X$ denotes the graded projective dimension of $X$, and gl.dim $B$ the (right) graded global homological dimension of $B$.

\subsection{Adams spectral sequence in triangulated categories}

Let $\mathcal{T}$ be a be a triangulated category (see eg, Gelfand and Manin [8, IV.1.1]) and $\operatorname{Hom}_{\mathcal{T}}(X, Y)_{*}$ denote $\operatorname{Hom}_{\mathcal{T}}\left(\Sigma^{*} X, Y\right)$, the graded Hom, for any $X, Y \in \mathcal{T}$. Recall the following definition.

Definition 2.2.1 Let $\mathcal{T}$ be a triangulated category with infinite coproducts. An object $P \in \mathcal{T}$ is compact if the functor $\operatorname{Hom}_{\mathcal{T}}(P,-)$ commutes with arbitrary coproducts. 
One says that $P$ is a compact generator if it is a compact object and in addition $\operatorname{Hom}_{\mathcal{T}}(P, X)_{*}=0$ implies that $X=0$, for any $X \in \mathcal{T}$ (or, equivalently, the functor $\operatorname{Hom}_{\mathcal{T}}(P,-)_{*}: \mathcal{T} \longrightarrow \operatorname{Grmod}-\operatorname{End}_{\mathcal{T}}(P)_{*}$ reflects isomorphisms $)$.

The following is well known (see eg, Benson, Krause and Schwede [1, Lemma 5.17 and Proposition A.4]). Although [1, Lemma 5.17] is stated for the derived category of a differential graded algebra, the proof given in [1] can be equally applied to general triangulated categories.

Lemma 2.2.2 Let $\mathcal{T}$ be a triangulated category with infinite coproducts and $P \in \mathcal{T}$ a compact generator.

(i) If $F \in \mathcal{T}$ and $\operatorname{Hom}_{\mathcal{T}}(P, F)_{*}$ is a projective $\operatorname{End}_{\mathcal{T}}(P)_{*}$-module, then the map $\operatorname{Hom}_{\mathcal{T}}(P,-)_{*}: \operatorname{Hom}_{\mathcal{T}}(F, X) \longrightarrow \operatorname{Hom}_{\operatorname{End}}(P)_{*}\left(\operatorname{Hom}_{\mathcal{T}}(P, F)_{*}, \operatorname{Hom}_{\mathcal{T}}(P, X)_{*}\right)$ is an isomorphism for any $X \in \mathcal{T}$.

(ii) For any projective graded $\operatorname{End}_{\mathcal{T}}(P)_{*}$-module $M$, there exists $G \in \mathcal{T}$ such that

$$
\operatorname{Hom}_{\mathcal{T}}(P, G)_{*} \cong M
$$

in $\operatorname{Grmod}-\operatorname{End}_{\mathcal{T}}(P)_{*}$.

Next, using Lemma 2.2.2, one gets the following proposition.

Proposition 2.2.3 (The Adams spectral sequence) Suppose $\mathcal{T}$ is a triangulated category with infinite coproducts and $P \in \mathcal{T}$ a compact generator, and assume that the graded global homological dimension of $\operatorname{End}_{\mathcal{T}}(P)_{*}$ is finite. Then there is a bounded convergent spectral sequence

$$
E_{2}^{p q}=\operatorname{Ext}_{\operatorname{End}}^{p(P)_{*}}\left(\operatorname{Hom}_{\mathcal{T}}(P, X)_{*}, \operatorname{Hom}_{\mathcal{T}}(P, Y)_{*}[q]\right) \Rightarrow \operatorname{Hom}_{\mathcal{T}}\left(X, \Sigma^{p+q} Y\right) .
$$

In fact, Proposition 2.2.3 is a special case of Christensen $[4,4.5]$.

\subsection{Model categories}

In this subsection we recall some basic properties of model categories and simplicial model categories.

A model category $\mathcal{M}$ is a bicomplete category equipped with three classes of morphisms called weak equivalences, fibrations and cofibrations, satisfying certain axioms. We will not list these axioms here. The point of this structure is that it allows one to "do 
homotopy theory" in $\mathcal{M}$. Good references for model categories include Dwyer and Spaliński [5], Hovey [12] and Quillen [18].

The fundamental example of a model category is the category sSet of simplicial sets (see Quillen [18] and Goerss and Jardine [9, 1.11.3]). Further important examples are the category Top of topological spaces (see [18] and [12, 2.4.19]) and the category $\mathrm{Ch}(K)$ of chain complexes of modules over a ring $K[12,2.3 .11]$.

For any model category $\mathcal{M}$, one has the associated homotopy category $\operatorname{Ho}(\mathcal{M})$ which is defined as the localization of $\mathcal{M}$ with respect to the class of weak equivalences (see eg, $[12,1.2]$ or [5]). The model structure guarantees that we do not face set theoretic problems when passing to localization, ie, $\operatorname{Ho}(\mathcal{M})$ has $\operatorname{Hom}-$ sets. Note that $\operatorname{Ho}(\mathcal{M})$ admits other equivalent descriptions as well. For example, $\operatorname{Ho}(\mathcal{M})$ is equivalent to the homotopy category of cofibrant objects. More precisely, let $\mathcal{M}_{\text {cof }}$ denote the full subcategory of cofibrant objects in $\mathcal{M}$. Then $\operatorname{Ho}(\mathcal{M})$ is equivalent to the localization $\mathcal{M}_{\text {cof }}\left[\mathcal{W}^{-1}\right]$, where $\mathcal{W}$ is the class of weak equivalences in $\mathcal{M}_{\text {cof }}$. This description of the homotopy category is most convenient one for our exposition, and therefore we make the following convention.

Convention 2.3.1 In what follows, we let $\operatorname{Ho}(\mathcal{M})$ denote the category $\mathcal{M}_{\text {cof }}\left[\mathcal{W}^{-1}\right]$.

Note that the class $\mathcal{W}$ admits a calculus of left fractions [18]. This is one of the advantages of Convention 2.3.1.

Further, recall the definition of a Quillen adjunction.

Definition 2.3.2 A Quillen adjunction between two model categories $\mathcal{M}$ and $\mathcal{N}$ is a pair of adjoint functors

$$
F: \mathcal{M} \rightleftarrows \mathcal{N}: G,
$$

where the left adjoint $F$ preserves cofibrations and acyclic cofibrations (or, equivalently, $G$ preserves fibrations and acyclic fibrations).

We refer to $F$ as a left Quillen functor and to $G$ as a right Quillen functor. Quillen's total derived functor theorem (see eg, [18] or [9, 2.8.7]) says that any such pair of adjoint functors induces an adjunction

$$
\mathbf{L} F: \operatorname{Ho}(\mathcal{M}) \rightleftarrows \operatorname{Ho}(\mathcal{N}): \mathbf{R} G .
$$

The functor $\mathbf{L} F$ is called the left derived functor of $F$ and $\mathbf{R} G$ the right derived functor of $G$. If $\mathbf{L} F$ is an equivalence of categories (or, equivalently, $\mathbf{R} G$ is an equivalence), then the Quillen adjunction is called a Quillen equivalence. 
Convention 2.3.1 allows us to give a very simple description of $\mathbf{L} F$. Indeed, the functor

$$
\left.F\right|_{\mathcal{M}_{\text {cof }}}: \mathcal{M}_{\text {cof }} \longrightarrow \mathcal{N}_{\text {cof }}
$$

preserves weak equivalences [12, 1.1.12] and, therefore, it induces a functor between the localizations. This functor is exactly $\mathbf{L} F$ in terms of Convention 2.3.1.

An important class of model categories is the class of simplicial model categories as in $[9,2.3]$. These are model categories which are enriched, tensored and cotensored over sSet and which satisfy Quillen's axiom SM7 [9, 2.3.1] (or, equivalently, the pushout product axiom $[9,2.3 .11]$ ). If a simplicial model category $\mathcal{M}$ is pointed, ie, the terminal object is isomorphic to the initial one, then $\mathcal{M}$ is enriched over the category sSet $_{*}$ of pointed simplicial sets. In particular, we have the functors

$$
-\wedge-: \mathbf{s S e t}_{*} \times \mathcal{M} \longrightarrow \mathcal{M}, \quad \operatorname{Map}_{\mathcal{M}}(-,-): \mathcal{M}^{\mathrm{op}} \times \mathcal{M} \longrightarrow \mathbf{s S e t}_{*}
$$

and the adjunction

$$
\operatorname{Hom}_{\mathcal{M}}(K \wedge X, Y) \cong \operatorname{Hom}_{\text {sSet }_{*}}\left(K, \operatorname{Map}_{\mathcal{M}}(X, Y)\right) .
$$

Remark 2.3.3 It follows from the axioms of pointed simplicial (model) categories that for any $n \geq 0$ and $X, Y \in \mathcal{M}$, there is a canonical natural isomorphism

$$
\operatorname{Map}_{\mathcal{M}}(X, Y)_{n} \cong \operatorname{Hom}_{\mathcal{M}}\left(\Delta[n]_{+} \wedge X, Y\right)
$$

where $\Delta[n]_{+}$is the union of $\Delta[n]$ with a disjoint base point. For any element $\theta$ in $\operatorname{Map}_{\mathcal{M}}(X, Y)_{n}$, the corresponding morphism $\Delta[n]_{+} \wedge X \longrightarrow Y$ will be denoted again by $\theta$.

\subsection{Stable model categories}

Recall from [18] (see also [12, 6.1.1]) that the homotopy category $\operatorname{Ho}(\mathcal{M})$ of a pointed model category $\mathcal{M}$ supports a suspension functor

$$
\Sigma: \operatorname{Ho}(\mathcal{M}) \longrightarrow \operatorname{Ho}(\mathcal{M})
$$

with a right adjoint loop functor

$$
\Omega: \operatorname{Ho}(\mathcal{M}) \longrightarrow \operatorname{Ho}(\mathcal{M}) .
$$

Definition 2.4.1 A stable model category is a pointed model category for which the functors $\Sigma$ and $\Omega$ are inverse equivalences. 
Remark 2.4.2 If $\mathcal{M}$ is a pointed simplicial model category, then the suspension functor

$$
\Sigma: \operatorname{Ho}(\mathcal{M}) \longrightarrow \operatorname{Ho}(\mathcal{M})
$$

admits a very simple description. Indeed, by the simplicial model category axioms the functor

$$
S^{1} \wedge-: \mathcal{M} \longrightarrow \mathcal{M}
$$

is a left Quillen functor. Then $\Sigma$ can be defined as the left derived functor of $S^{1} \wedge$.

Note that if $\mathcal{M}$ is stable, then the homotopy category $\operatorname{Ho}(\mathcal{M})$ is a triangulated category $[12,7.1 .6]$ with $\Sigma$ a shift functor. We will now recall the construction of distinguished triangles in $\operatorname{Ho}(\mathcal{M})$ in the case when $\mathcal{M}$ is simplicial.

Definition 2.4.3 Suppose $\mathcal{M}$ is a simplicial stable model category and $f: X \longrightarrow Y$ a morphism in $\mathcal{M}_{\text {cof }}$. The pushout of $f$ along the morphism

$$
\text { incl } \wedge 1: X \cong S^{0} \wedge X \longrightarrow(I, 0) \wedge X
$$

is called the mapping cone of $f$ and is denoted by Cone $(f)$.

Thus Cone $(f)$ comes with the pushout square

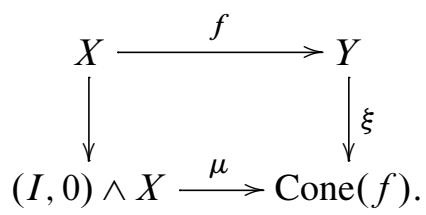

Furthermore, the universal property of pushout implies that there is a commutative diagram

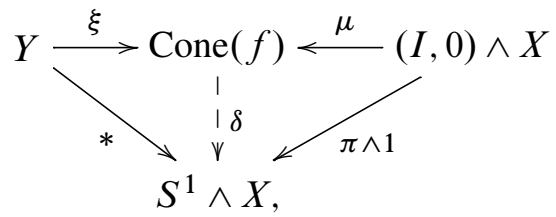

where $\pi: I \longrightarrow S^{1}$ is the projection.

Definition 2.4.4 Let $\mathcal{M}$ be a simplicial stable model category and $f: X \longrightarrow Y$ a morphism in $\mathcal{M}_{\text {cof }}$. The elementary triangle associated to $f$ is the triangle

$$
X \stackrel{f}{\longrightarrow} Y \stackrel{\xi}{\longrightarrow} \text { Cone }(f) \stackrel{\delta}{\longrightarrow} S^{1} \wedge X .
$$


A triangle

$$
A \longrightarrow B \longrightarrow C \longrightarrow \Sigma A
$$

in $\operatorname{Ho}(\mathcal{M})$ is called distinguished if it is isomorphic to an elementary one.

The distinguished triangles together with $\Sigma$ define the triangulated structure on $\operatorname{Ho}(\mathcal{M})$. Now let us give some examples of stable model categories which are important in what follows.

Example 2.4.5 For any ring $K$, the category $\mathrm{Ch}(K)$ of unbounded chain complexes of $K$-modules with the projective model structure is a stable model category [12, 2.3.11]. The weak equivalences and fibrations in this model structure are quasi-isomorphisms (ie, homology isomorphisms) and degreewise epimorphisms, respectively.

Example 2.4.6 Let $R$ be a symmetric ring spectrum. The category Mod $-R$ of right $R$-modules admits a stable simplicial model structure; see Hovey, Shipley and Smith [13, Corollary 5.5.2]. The homotopy category $\operatorname{Ho}(\operatorname{Mod}-R)$ is called the derived category of $R$, denoted by $\mathcal{D}(R)$. Note that $R$ is a compact generator of $\mathcal{D}(R)$ and the representable homological functor (see eg, [8, IV.1.6]) associated to $R$ is the homotopy group functor $\pi_{*}: \mathcal{D}(R) \longrightarrow \mathbf{G r m o d}-\pi_{*} R$.

Example 2.4.7 Let $A$ be a differential graded ring. Then, by Example 2.4.5 and Schwede and Shipley [21, 4.1], the category Mod- $A$ of differential graded right modules over $A$ has a projective model structure in which the weak equivalences and fibrations are as in Example 2.4.5. This model structure is stable as well. The category $\operatorname{Ho}(\operatorname{Mod}-A)$ is called the derived category of $A$, denoted by $\mathcal{D}(A)$. The differential graded module $A$ is a compact generator of $\mathcal{D}(A)$ and the representable homological functor associated to $A$ is the homology $H_{*}: \mathcal{D}(A) \longrightarrow \mathbf{G r m o d}-H_{*} A$.

By Shipley [22, 2.15], there is a symmetric ring spectrum $H A$ such that Mod- $H A$ is Quillen equivalent to $\operatorname{Mod}-A$. In particular, the derived category $\mathcal{D}(A)$ is triangulated equivalent to $\mathcal{D}(H A)$. Thus, from the point of view of model category theory Example 2.4.7 is a special case of Example 2.4.6.

Remark 2.4.8 Note that the triangulated structure on the derived category $\mathcal{D}(A)$ of a differential graded ring $A$, comes from the usual shift functor and algebraic mapping cone construction. More precisely, for any $M \in \mathcal{D}(A)$, the suspension of $M$, denoted by $M[1]$, is given by

$$
M[1]_{n}=M_{n-1}, \quad d_{n}^{M[1]}=-d_{n-1}^{M} .
$$


Obviously, $M[1]$ inherits the structure of a graded right $A$-module from that of $M$ (the action of $A$ on $M[1]$ is not twisted by a sign). Furthermore, it is clear that $d^{M[1]}$ satisfies the Leibniz rule.

Next, let $f: M \longrightarrow M^{\prime}$ be a morphism of DG modules over $A$. The (algebraic) mapping cone $C(f)$ of $f$ is defined by

$$
\begin{aligned}
(C(f))_{n} & =M_{n-1} \oplus M_{n}^{\prime}, \quad n \in \mathbb{Z}, \\
d\left(m, m^{\prime}\right) & =\left(-d m, f(m)+d m^{\prime}\right) .
\end{aligned}
$$

Clearly, $C(f)$ inherits the structure of graded (right) $A$-module from that of $M$ and $M^{\prime}$ and the differential of $C(f)$ satisfies the Leibniz rule. Thus $(C(f), d)$ is a DG $A$-module. Further, there are canonical morphisms of DG modules

$$
\begin{aligned}
& \iota: M^{\prime} \longrightarrow C(f), \quad \iota\left(m^{\prime}\right)=\left(0, m^{\prime}\right), \\
& \text { д: } C(f) \longrightarrow M[1], \quad \partial\left(m, m^{\prime}\right)=m .
\end{aligned}
$$

These morphisms together with $f: M \longrightarrow M^{\prime}$ form a triangle

$$
M \stackrel{f}{\longrightarrow} M^{\prime} \stackrel{\iota}{\longrightarrow} C(f) \stackrel{\partial}{\longrightarrow} M[1],
$$

called the elementary triangle associated to $f$.

A triangle in $\mathcal{D}(A)$ is distinguished if and only if it is isomorphic to an elementary one.

\subsection{Diagram categories}

We will use model structures on diagram categories throughout the paper. Therefore, we recall some facts and notions concerning them.

Definition 2.5.1 $[12,5.1 .1]$ Let $\omega$ denote the poset category of the ordered set $\{0,1,2, \ldots\}$. A small category $\mathcal{C}$ is called a direct category if there exists a functor $f: \mathcal{C} \longrightarrow \omega$ that sends nonidentity morphisms to nonidentity morphisms.

Definition 2.5.2 $[12,5.1 .2]$ Suppose $\mathcal{M}$ is a category with small colimits, $\mathcal{C}$ a small category, $z$ an object in $\mathcal{C}$ and $\mathcal{C}_{z}$ the category of all nonidentity morphisms in $\mathcal{C}$ with codomain $z$. The latching space functor $L_{z}: \mathcal{M}^{\mathcal{C}} \longrightarrow \mathcal{M}$ is the composition

$$
\mathcal{M}^{\mathcal{C}} \longrightarrow \mathcal{M}^{\mathcal{C}} \stackrel{\text { colim }}{\longrightarrow} \mathcal{M}
$$

where the first arrow is restriction. Note that we have a natural transformation

$$
L_{z} X \longrightarrow X_{z}
$$

for any fixed object $z \in \mathcal{C}$. 
The following proposition is proved in [12, 5.1.3].

Proposition 2.5.3 Given a model category $\mathcal{M}$ and a direct category $\mathcal{C}$, there is a model structure on $\mathcal{M}^{\mathcal{C}}$ in which a morphism of diagrams $f: X \longrightarrow Y$ is a weak equivalence (resp. fibration) if and only if the map $f_{z}: X_{z} \longrightarrow Y_{z}$ is a weak equivalence (resp. fibration) for all $z \in \mathcal{C}$. Furthermore, $f: X \longrightarrow Y$ is an (acyclic) cofibration if and only if the induced map $X_{z} \bigvee_{L_{z} X} L_{z} Y \longrightarrow Y_{z}$ is an (acyclic) cofibration for all $z \in \mathcal{C}$.

Example 2.5.4 Any finite poset $\mathcal{P}$ is a direct category. Therefore, for any model category $\mathcal{M}$, we have the model structure of Proposition 2.5.3 on $\mathcal{M}^{\mathcal{P}}$.

Example 2.5.5 Suppose $\mathcal{M}$ is a model category and $N \geq 2$ a natural number. Let $\mathcal{C}_{N}$ denote the poset consisting of elements $\left\{\beta_{i}, \zeta_{i} \mid i \in \mathbb{Z} / N \mathbb{Z}\right\}$ such that $\beta_{i}<\zeta_{i}$ and $\beta_{i}<\zeta_{i+1}$, ie,

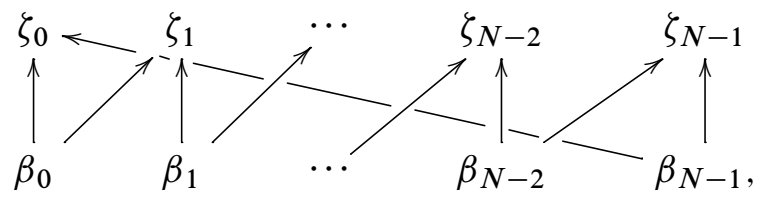

and consider $X \in \mathcal{M}^{\mathcal{C}_{N}}$. Then, by Proposition 2.5.3, $X$ is cofibrant if and only if the canonical map

$$
L_{z} X \longrightarrow X_{z}
$$

is a cofibration in $\mathcal{M}$ for all $z \in \mathcal{C}_{N}$, ie, if and only if $X_{\beta_{i}}$ is cofibrant and the induced morphism $X_{\beta_{i-1}} \vee X_{\beta_{i}} \longrightarrow X_{\zeta_{i}}$ is a cofibration, for any $i \in \mathbb{Z} / N \mathbb{Z}$.

Example 2.5.6 The category

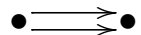

is a direct category. Thus, by Proposition 2.5.3, we get a model structure on the category of parallel arrows in $\mathcal{M}$.

It follows from $[12,5.1 .5]$ that for any model category $\mathcal{M}$ and direct category $\mathcal{C}$, there is a Quillen adjunction

$$
\text { colim: } \mathcal{M}^{\mathcal{C}} \longleftrightarrow \mathcal{M}: \text { const, }
$$

where colim is a left Quillen functor. 
Definition 2.5.7 The left derived functor of colim: $\mathcal{M}^{\mathcal{C}} \longrightarrow \mathcal{M}$ is called the homotopy colimit, denoted by

$$
\text { Hocolim: } \operatorname{Ho}\left(\mathcal{M}^{\mathcal{C}}\right) \longrightarrow \operatorname{Ho}(\mathcal{M}) \text {. }
$$

If $\mathcal{C}$ is as in Example 2.5.6, then the homotopy colimit is called the homotopy coequalizer.

Remark 2.5.8 Let $\mathcal{C}$ be a direct category (Definition 2.5.1). If $\mathcal{M}$ is a simplicial model category, then so is $\mathcal{M}^{\mathcal{C}}$. Indeed, the mapping spaces for $\mathcal{M}^{\mathcal{C}}$ are given by the end construction

$$
\operatorname{Map}_{\mathcal{M}^{\mathcal{C}}}(X, Y)=\int_{z \in \mathcal{C}} \operatorname{Map}_{\mathcal{M}}\left(X_{z}, Y_{z}\right)
$$

see Mac Lane [17, IX.5]. The tensors and cotensors are defined levelwise.

\subsection{Long exact sequences}

In this subsection we recall the Mayer-Vietoris sequence of a homotopy pullback and the long exact sequence of a homotopy coequalizer.

Lemma 2.6.1 (Dyer and Roitberg [6]) Let

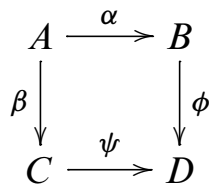

be a homotopy pullback square of pointed simplicial sets. Then there is a long exact Mayer-Vietoris sequence

$$
\begin{aligned}
& \cdots \rightarrow \pi_{n}(A) \stackrel{\left(\alpha_{*}, \beta_{*}\right)}{\longrightarrow} \pi_{n}(B) \times \pi_{n}(C) \stackrel{\left(\phi_{*},-\psi_{*}\right)}{\longrightarrow} \pi_{n}(D) \stackrel{\partial}{\rightarrow} \pi_{n-1}(A) \rightarrow \cdots \\
& \cdots \rightarrow \pi_{1}(A) \stackrel{\left(\alpha_{*}, \beta_{*}\right)}{\longrightarrow} \pi_{1}(B) \times \pi_{1}(C) \stackrel{\left(\phi_{*}, \psi_{*}^{-1}\right)}{\longrightarrow} \pi_{1}(D) \stackrel{\partial}{\rightarrow} \pi_{0}(A) \stackrel{\left(\alpha_{*}, \beta_{*}\right)}{\longrightarrow} \pi_{0}(B) \times \pi_{0}(C) .
\end{aligned}
$$

Note that if

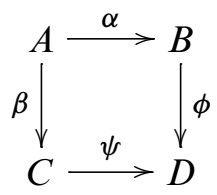

is a pullback square of Kan simplicial sets and $\phi$ a Kan fibration, then it is in fact a homotopy pullback. In this case the connecting homomorphism

$$
\text { ว: } \pi_{n}(D) \longrightarrow \pi_{n-1}(A)
$$


can be described as follows.

Lemma 2.6.2 Suppose that

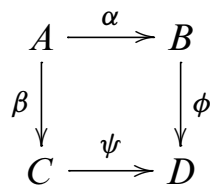

is a pullback square of pointed Kan simplicial sets, and $\phi$ a Kan fibration. Suppose further that $F$ is the fiber of $\phi$ and $\beta$, and $\varepsilon: F \longrightarrow A$ the inclusion. Then the connecting homomorphism

$$
\partial: \pi_{n}(D) \longrightarrow \pi_{n-1}(A)
$$

of the Mayer-Vietoris sequence is given by the composite

$$
\pi_{n}(D) \stackrel{\partial^{\prime}}{\longrightarrow} \pi_{n-1}(F) \stackrel{\varepsilon_{*}}{\longrightarrow} \pi_{n-1}(A),
$$

where $\partial^{\prime}$ is the connecting homomorphism of the long exact sequence of the fibration $\phi: B \longrightarrow D$; see $\operatorname{Kan}[14,3.5]$.

\section{Lemma 2.6.3 Let}

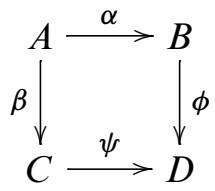

be a homotopy pullback square of simplicial sets with $A, B, C, D$ homotopy associative and homotopy commutative $H$-spaces, and $\alpha, \beta, \phi, \psi H$-space maps. Then the long exact Mayer-Vietoris sequence of this square can be extended to the right by one further term. More precisely, there is a long exact sequence of abelian groups

$$
\begin{aligned}
& \cdots \rightarrow \pi_{n}(A) \stackrel{\left(\alpha_{*}, \beta_{*}\right)}{\longrightarrow} \pi_{n}(B) \oplus \pi_{n}(C) \stackrel{\left(\phi_{*},-\psi_{*}\right)}{\longrightarrow} \pi_{n}(D) \stackrel{\partial}{\rightarrow} \pi_{n-1}(A) \rightarrow \cdots \\
& \cdots \rightarrow \pi_{1}(B) \oplus \pi_{1}(C) \stackrel{\left(\phi_{*},-\psi_{*}\right)}{\longrightarrow} \pi_{1}(D) \stackrel{\partial}{\rightarrow} \\
& \pi_{0}(A) \stackrel{\left(\alpha_{*}, \beta_{*}\right)}{\longrightarrow} \pi_{0}(B) \oplus \pi_{0}(C) \stackrel{\left(\phi_{*},-\psi_{*}\right)}{\longrightarrow} \pi_{0}(D) .
\end{aligned}
$$

Next we recall the following lemma.

Lemma 2.6.4 Let $\mathcal{M}$ be a stable model category and

$$
X \underset{g}{\stackrel{f}{\longrightarrow}} Y \stackrel{h}{\longrightarrow} Z
$$


a coequalizer diagram in $\mathcal{M}$. Suppose that $X$ is cofibrant and

$$
(f, g): X \vee X \longrightarrow Y
$$

is a cofibration (ie, the given coequalizer is a homotopy coequalizer). Then there is a morphism $\delta: Z \longrightarrow \Sigma X$ in $\operatorname{Ho}(\mathcal{M})$ such that the triangle

$$
X \stackrel{f-g}{\longrightarrow} Y \stackrel{h}{\longrightarrow} Z \stackrel{\delta}{\longrightarrow} \Sigma X
$$

is distinguished.

This lemma essentially follows from the remark at the end of Section I.4 in Brown [3].

Corollary 2.6.5 Let $\mathcal{M}$ be a stable model category, $\mathcal{A}$ an abelian category and $F: \operatorname{Ho}(\mathcal{M}) \longrightarrow \mathcal{A}$ a homological functor. Then any homotopy coequalizer

$$
X \underset{g}{\stackrel{f}{\longrightarrow}} Y \stackrel{h}{\longrightarrow} Z
$$

in $\mathcal{M}$ induces a long exact sequence

$$
\cdots \rightarrow F(X) \stackrel{F(f)-F(g)}{\longrightarrow} F(Y) \stackrel{F(h)}{\longrightarrow} F(Z) \stackrel{\partial}{\rightarrow} F(\Sigma X) \rightarrow \cdots .
$$

\section{Franke's functor}

\subsection{Formulation of main results}

Let $\mathcal{M}$ be a stable model category and $[X, Y]$ denote the $\operatorname{Hom}_{\text {-group }} \operatorname{Hom}_{\mathrm{Ho}(\mathcal{M})}(X, Y)$, for any $X, Y \in \operatorname{Ho}(\mathcal{M})$. Next, suppose $P \in \operatorname{Ho}(\mathcal{M})$ is a compact generator. In the sequel we use the notation

$$
\pi_{*} X=[P, X]_{*}=\operatorname{Hom}_{\mathrm{Ho}(\mathcal{M})}\left(\Sigma^{*} P, Y\right) .
$$

In particular, $\pi_{*} P$ denotes the graded ring $[P, P]_{*}$.

Remark 3.1.1 The suspension functor $\Sigma: \operatorname{Ho}(\mathcal{M}) \longrightarrow \operatorname{Ho}(\mathcal{M})$ induces a natural isomorphism of graded $\pi_{*} P$-modules

$$
\pi_{*}(\Sigma X) \cong\left(\pi_{*} X\right)[1] .
$$

Having this in mind, we identify $\pi_{*}(\Sigma X)$ with $\left(\pi_{*} X\right)[1]$ in what follows.

Definition 3.1.2 Suppose $N \geq 2$ is a natural number. A graded ring $B$ is said to be $N$-sparse if it is concentrated in degrees divisible by $N$. 
Definition 3.1.3 Let $\mathcal{M}$ be a stable model category and $P \in \mathcal{M}$ a compact generator. We say that an object $X \in \operatorname{Ho}(\mathcal{M})$ is $k$-dimensional (with respect to $P$ ) if the projective dimension of $\pi_{*} X$ in $\mathbf{G r m o d}-\pi_{*} P$ is equal to $k$.

Now we are ready to list the main results and their corollaries.

Theorem 3.1.4 Let $\mathcal{M}$ be a stable model category and $P$ a fixed compact generator of $\operatorname{Ho}(\mathcal{M})$. Suppose that the graded ring $\pi_{*} P$ is $N$-sparse and the (right) graded global homological dimension of $\pi_{*} P$ is less than $N$. Then one can construct a functor

$$
\mathscr{R}: \mathcal{D}\left(\pi_{*} P\right) \longrightarrow \operatorname{Ho}(\mathcal{M})
$$

such that the following hold:

(i) The diagram

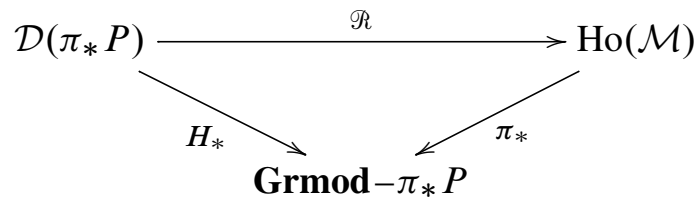

commutes up to a natural isomorphism.

(ii) The functor $\mathscr{R}$ restricts to an equivalence of the full subcategories of at most one-dimensional objects.

(iii) Let $X \in \operatorname{Ho}(\mathcal{M})$ and suppose that $X$ has dimension at most two. Then there exists $M \in \mathcal{D}\left(\pi_{*} P\right)$ such that $\mathscr{R}(M)$ is isomorphic to $X$ in $\operatorname{Ho}(\mathcal{M})$.

Theorem 3.1.5 Let $\mathcal{M}$ be a stable model category and $P$ a compact generator of $\operatorname{Ho}(\mathcal{M})$. Suppose that the graded ring $\pi_{*} P$ is $N$-sparse and the (right) graded global homological dimension of $\pi_{*} P$ is less than $N-1$. Then the functor

$$
\mathscr{R}: \mathcal{D}\left(\pi_{*} P\right) \longrightarrow \operatorname{Ho}(\mathcal{M})
$$

restricts to an equivalence of the full subcategories of at most two dimensional objects.

In particular, we have the following corollary.

Corollary 3.1.6 Let $\mathcal{M}$ be a stable model category and $P$ a compact generator of $\operatorname{Ho}(\mathcal{M})$. Suppose that the graded ring $\pi_{*} P$ is $N$-sparse, the (right) graded global homological dimension of $\pi_{*} P$ is equal to two and $N \geq 4$. Then the functor

$$
\mathscr{R}: \mathcal{D}\left(\pi_{*} P\right) \longrightarrow \operatorname{Ho}(\mathcal{M})
$$

is an equivalence of categories. 
Theorems 1.1.1 and 1.1.2 are obtained as special cases of Theorems 3.1.4 and 3.1.5, respectively. Further, in view of Example 2.4.7, one has the following corollaries.

Corollary 3.1.7 Let $A$ be a differential graded ring. Suppose that the graded homology ring $H_{*} A$ is concentrated in dimensions divisible by a natural number $N$ and assume that the (right) graded global homological dimension of $H_{*} A$ is less than $N$. Then one can construct a functor

$$
\mathscr{R}: \mathcal{D}\left(H_{*} A\right) \longrightarrow \mathcal{D}(A)
$$

such that the following hold:

(i) The diagram

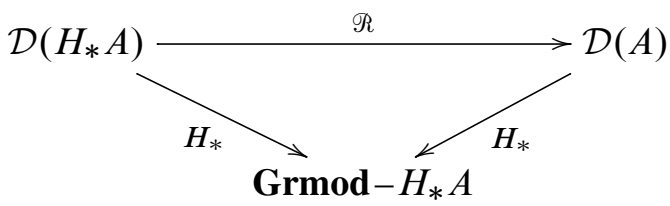

commutes up to a natural isomorphism.

(ii) The functor $\mathscr{R}: \mathcal{D}\left(H_{*} A\right) \longrightarrow \mathcal{D}(A)$ restricts to an equivalence of the full subcategories of at most one-dimensional objects.

(iii) Let $X \in \mathcal{D}(A)$ and suppose $X$ has dimension at most two. Then there exists $M \in \mathcal{D}\left(H_{*} A\right)$ such that $\mathscr{R}(M)$ is isomorphic to $X$ in $\mathcal{D}(A)$.

Corollary 3.1.8 Let $A$ be a differential graded ring. Suppose that the graded homology ring $H_{*} A$ is $N$-sparse and the (right) graded global homological dimension of $H_{*} A$ is less than $N-1$. Then the functor

$$
\mathscr{R}: \mathcal{D}\left(H_{*} A\right) \longrightarrow \mathcal{D}(A)
$$

restricts to an equivalence of the full subcategories of at most two-dimensional objects.

Corollary 3.1.9 Let $A$ be a differential graded ring. Suppose that the graded homology ring $H_{*} A$ is $N$-sparse, the (right) graded global homological dimension of $H_{*} A$ is equal to two and $N \geq 4$. Then the functor

$$
\mathscr{R}: \mathcal{D}\left(H_{*} A\right) \longrightarrow \mathcal{D}(A)
$$

is an equivalence of categories.

The functor $\mathscr{R}$ is constructed in Subsection 3.4 and the proof of Theorem 3.1.4(i) is given in Subsection 3.4. Next, Theorem 3.1.4(ii) is proved in Section 5, and finally, the proofs of Theorems 3.1.4(iii) and 3.1.5 are discussed in Section 6. 


\subsection{Some consequences of the Adams spectral sequence}

In this subsection we state some known facts which follow from the Adams spectral sequence of Proposition 2.2.3,

$$
E_{2}^{p q}=\operatorname{Ext}_{\pi_{*} P}^{p}\left(\pi_{*} X, \pi_{*} Y[q]\right) \Rightarrow\left[X, \Sigma^{p+q} Y\right]
$$

Let $\mathcal{M}, P$ and $N$ be as in Theorem 3.1.4. Then the category of graded $\pi_{*} P$-modules splits as follows:

$$
\text { Grmod }-\pi_{*} P \sim \mathcal{B} \oplus \mathcal{B}[1] \oplus \cdots \oplus \mathcal{B}[N-1]
$$

where $\mathcal{B}$ is the full subcategory of $\mathbf{G r m o d}-\pi_{*} P$ consisting of all those modules which are concentrated in degrees divisible by $N$.

Let $\mathcal{E}_{i}$ denote the full subcategory of $\operatorname{Ho}(\mathcal{M})$ consisting of objects $X \in \operatorname{Ho}(\mathcal{M})$ with

$$
\pi_{*} X \in \mathcal{B}[i] .
$$

Proposition 3.2.1 $\quad$ (i) The functor

$$
\left.\pi_{*}\right|_{\mathcal{E}_{i}}: \mathcal{E}_{i} \longrightarrow \mathcal{B}[i]
$$

is an equivalence of categories. In particular, for any $i \in \mathbb{Z} / N \mathbb{Z}$ and $X, Y \in \mathcal{E}_{i}$, the natural map

$$
\pi_{*}:[X, Y] \longrightarrow \operatorname{Hom}_{\pi_{*} P}\left(\pi_{*} X, \pi_{*} Y\right)
$$

is an isomorphism.

(ii) For any $i \in \mathbb{Z} / N \mathbb{Z}, X \in \mathcal{E}_{i-1}$ and $Y \in \mathcal{E}_{i}$, there is a natural isomorphism

$$
[X, Y] \cong \operatorname{Ext}_{\pi_{*} P}^{1}\left(\pi_{*} X[1], \pi_{*} Y\right)
$$

(iii) For any $i \in \mathbb{Z} / N \mathbb{Z}$ and $X, Y \in \mathcal{E}_{i}$, there is a natural isomorphism

$$
[\Sigma X, Y] \cong \operatorname{Ext}_{\pi_{*} P}^{N-1}\left(\pi_{*} X[N], \pi_{*} Y\right) .
$$

This proposition immediately follows from the Adams spectral sequence. (The conditions in Theorem 3.1.4 imply that the corresponding spectral sequences collapse at $E_{2}$ and all appropriate terms vanish.) Note that here one essentially uses that the graded global homological dimension of $\pi_{*} P$ is less than $N$. Indeed, all $\operatorname{Ext}_{\pi_{*}}^{i} P^{- \text {groups for }}$ $i \geq N$ vanish and hence, $\operatorname{Ext}_{\pi_{*} P}^{N k}\left(\pi_{*} X[N k], \pi_{*} Y\right)=0$, for $k>0, X, Y \in \mathcal{E}_{i}$, which implies Proposition 3.2.1(i). Similar arguments yield the proofs of (ii) and (iii). 
Remark 3.2.2 If $X \in \mathcal{E}_{i-1}$ and $Y \in \mathcal{E}_{i}$ then $\pi_{*} f=0$ for any $f \in[X, Y]$. This and the construction of the Adams spectral sequence enables one to get an explicit description of the isomorphism in Proposition 3.2.1(ii). Indeed, for any $f \in[X, Y]$, a distinguished triangle

$$
X \stackrel{f}{\longrightarrow} Y \stackrel{\xi}{\longrightarrow} \text { Cone }(k) \stackrel{\delta}{\longrightarrow} \Sigma X
$$

gives a short exact sequence

$$
E: 0 \longrightarrow \pi_{*} Y \stackrel{\pi_{*} \xi}{\longrightarrow} \pi_{*} \operatorname{Cone}(k) \stackrel{\pi_{*} \delta}{\longrightarrow} \pi_{*} X[1] \longrightarrow 0,
$$

and the isomorphism of Proposition 3.2.1(ii) sends $f$ to the extension class of $E$.

\subsection{Crowned diagrams and DG modules}

In this subsection we start with the proof of Theorem 3.1.4. In order to simplify the exposition, the model category $\mathcal{M}$ will be assumed to be simplicial in the sequel. (Note that our examples of model categories (see Theorem 1.1.1 and Corollary 3.1.7) are in fact simplicial or Quillen equivalent to simplicial ones.) However, the proof can be applied for stable model categories without any enrichment as well, using the techniques of cosimplicial frames from [12] (see Subsection 4.1 for details).

Let $\mathcal{C}_{N}$ be as in Example 2.5.5. Suppose $X$ is an object of $\left(\mathcal{M}^{\mathcal{C}_{N}}\right)_{\text {cof }}$ and

$$
l_{i}: X_{\beta_{i}} \longrightarrow X_{\zeta_{i}}, \quad k_{i}: X_{\beta_{i-1}} \longrightarrow X_{\zeta_{i}}, \quad i \in \mathbb{Z} / N \mathbb{Z}
$$

the morphisms of $X$. Since $X$ is cofibrant in $\mathcal{M}^{\mathcal{C}_{N}}$, the objects $X_{\beta_{i}}$ and $X_{\zeta_{i}}$ are cofibrant in $\mathcal{M}, i \in \mathbb{Z} / N \mathbb{Z}$. Let

$$
\begin{gathered}
Z^{(i)}(X)=\pi_{*} X_{\zeta_{i}}, \quad B^{(i)}(X)=\pi_{*} X_{\beta_{i}}, \quad C^{(i)}(X)=\pi_{*} \text { Cone }\left(k_{i}\right), \\
\lambda^{(i)}=\pi_{*} l_{i}: B^{(i)}(X) \longrightarrow Z^{(i)}(X), \quad i \in \mathbb{Z} / N \mathbb{Z},
\end{gathered}
$$

where Cone $\left(k_{i}\right)$ denotes the cone construction from Definition 2.4.3 for simplicial model categories applied to $k_{i}$. Consider the mapping cone sequence

$$
X_{\beta_{i-1}} \stackrel{k_{i}}{\longrightarrow} X_{\zeta_{i}} \longrightarrow \text { Cone }\left(k_{i}\right) \longrightarrow \Sigma X_{\beta_{i-1}}
$$

of $k_{i}, i \in \mathbb{Z} / N \mathbb{Z}$. Applying the functor $\pi_{*}: \operatorname{Ho}(\mathcal{M}) \longrightarrow \operatorname{Grmod}-\pi_{*} P$ to the sequence $X_{\zeta_{i}} \longrightarrow$ Cone $\left(k_{i}\right) \longrightarrow \Sigma X_{\beta_{i-1}}$, we obtain the sequence

$$
Z^{(i)}(X) \stackrel{\iota^{(i)}}{\longrightarrow} C^{(i)}(X) \stackrel{\rho^{(i)}}{\longrightarrow} B^{(i-1)}(X)[1]
$$


see Remark 3.1.1. Clearly, $Z^{(i)}$ and $B^{(i)}$ are functors from $\left(\mathcal{M}^{\mathcal{C}_{N}}\right)$ cof to $\operatorname{Grmod}-\pi_{*} P$ and $\lambda^{(i)}$ is natural. Besides, since the cone construction of Definition 2.4.3 is functorial, $C^{(i)}$ is a functor from $\left(\mathcal{M}^{\mathcal{C}_{N}}\right)$ cof to $\mathbf{G r m o d}-\pi_{*} P$ as well, and $\iota^{(i)}$ and $\rho^{(i)}$ are natural. Further, $Z^{(i)}, B^{(i)}$ and $C^{(i)}$ pass through $\operatorname{Ho}\left(\mathcal{M}^{\mathcal{C}_{N}}\right)$ (as they send weak equivalences of diagrams to isomorphisms), and $\lambda^{(i)}, \iota^{(i)}, \rho^{(i)}$ induce natural transformations on the homotopy level. We use the same notation for the resulting functors and natural transformations.

Notation 3.3.1 let $\mathcal{L}$ denote the full subcategory of $\operatorname{Ho}\left(\mathcal{M}^{\mathcal{C}_{N}}\right)$ consisting of all those $X$ which satisfy the following conditions:

(i) The graded $\pi_{*} P$-modules $Z^{(i)}(X), B^{(i)}(X)$ are objects of $\mathcal{B}[i]$ for any $i$ $\in \mathbb{Z} / N \mathbb{Z}$.

(ii) The morphism $\lambda^{(i)}$ is a monomorphism for all $i \in \mathbb{Z} / N \mathbb{Z}$.

Now we are going to construct a functor

$$
\mathcal{Q}: \mathcal{L} \longrightarrow \operatorname{Mod}-\pi_{*} P .
$$

Let $X$ be an object of $\mathcal{L}$. As the functor

$$
\pi_{*}: \operatorname{Ho}(\mathcal{M}) \longrightarrow \text { Grmod }-\pi_{*} P
$$

is homological, the distinguished triangles

$$
X_{\beta_{i-1}} \stackrel{k_{i}}{\longrightarrow} X_{\zeta_{i}} \longrightarrow \operatorname{Cone}\left(k_{i}\right) \longrightarrow \Sigma X_{\beta_{i-1}}
$$

induce long exact sequences

$\cdots \longrightarrow B^{(i-1)}(X) \longrightarrow Z^{(i)}(X) \stackrel{\iota^{(i)}}{\longrightarrow} C^{(i)}(X) \stackrel{\rho^{(i)}}{\longrightarrow} B^{(i-1)}(X)[1] \longrightarrow Z^{(i)}(X)[1] \longrightarrow \cdots$.

Note that $B^{(i-1)}(X) \in \mathcal{B}[i-1]$ and $Z^{(i)}(X) \in \mathcal{B}[i]$ for all $i \in \mathbb{Z} / N \mathbb{Z}$, since $X \in \mathcal{L}$. Therefore, the morphisms $B^{(i-1)}(X) \longrightarrow Z^{(i)}(X)$ and $B^{(i-1)}(X)[1] \longrightarrow Z^{(i)}(X)[1]$ are zero. Consequently, for any $i \in \mathbb{Z} / N \mathbb{Z}$, we get a short exact sequence

$$
0 \longrightarrow Z^{(i)}(X) \stackrel{\iota^{(i)}}{\longrightarrow} C^{(i)}(X) \stackrel{\rho^{(i)}}{\longrightarrow} B^{(i-1)}(X)[1] \longrightarrow 0
$$

in Grmod $-\pi_{*} P$. Then, consider the following graded $\pi_{*} P$-modules

$$
\begin{aligned}
& C_{*}(X)=C^{(0)}(X) \oplus C^{(1)}(X) \oplus \cdots \oplus C^{(N-1)}(X), \\
& Z_{*}(X)=Z^{(0)}(X) \oplus Z^{(1)}(X) \oplus \cdots \oplus Z^{(N-1)}(X), \\
& B_{*}(X)=B^{(0)}(X) \oplus B^{(1)}(X) \oplus \cdots \oplus B^{(N-1)}(X) .
\end{aligned}
$$


The morphisms $\lambda^{(i)}, \iota^{(i)}, \rho^{(i)}, i \in \mathbb{Z} / N \mathbb{Z}$, induce morphisms between the direct sums

$$
\begin{aligned}
\lambda: B_{*}(X) \longrightarrow Z_{*}(X), & \lambda=\lambda^{(0)} \oplus \lambda^{(1)} \oplus \cdots \oplus \lambda^{(N-1)}, \\
\iota: Z_{*}(X) \longrightarrow C_{*}(X), & \iota=\iota^{(0)} \oplus \iota^{(1)} \oplus \cdots \oplus \iota^{(N-1)}, \\
\rho: C_{*}(X) \longrightarrow B_{*}(X)[1], & \rho=\rho^{(0)} \oplus \rho^{(1)} \oplus \cdots \oplus \rho^{(N-1)} .
\end{aligned}
$$

After summing up, we get a short exact sequence of $\pi_{*} P$-modules

$$
0 \longrightarrow Z_{*}(X) \stackrel{\iota}{\longrightarrow} C_{*}(X) \stackrel{\rho}{\longrightarrow} B_{*}(X)[1] \longrightarrow 0 .
$$

Splicing this short exact sequence with its shifted copy gives a differential graded $\pi_{*} P$-module. More precisely, define

$$
d=\iota[1] \lambda[1] \rho: C_{*}(X) \longrightarrow C_{*}(X)[1] .
$$

Then $d d=0$ and, therefore, we get a DG $\pi_{*} P$-module $\left(C_{*}(X), d\right)$. The desired functor,

$$
\mathcal{Q}: \mathcal{L} \longrightarrow \operatorname{Mod}-\pi_{*} P,
$$

is defined by $\mathcal{Q}(X)=\left(C_{*}(X), d\right)$.

Remark 3.3.3 Towards the end of this subsection, namely in 3.3.13, we will see that in many cases the functor $\mathcal{Q}$ is an equivalence of categories. In fact, the intuition behind the definition of $\mathcal{L}$ (Notation 3.3.1) is to construct diagram models that encode the structure of DG $\pi_{*}(P)$-modules. More precisely a diagram $X$ from $\mathcal{L}$ carries information about the differentials of the DG $\pi_{*}(P)$-module $\mathcal{Q}(X)$ and also remembers how $\mathcal{Q}(X)$ is built out of cycles and boundaries.

Remark 3.3.4 Let $\mathcal{L}^{\prime}$ denote the full subcategory of $\left(\mathcal{M}^{\mathcal{C}_{N}}\right)_{\text {cof }}$ consisting of all the objects $X$ with $Z^{(i)}(X), B^{(i)}(X) \in \mathcal{B}[i]$ and $\lambda^{(i)}$ a monomorphism, for any $i \in \mathbb{Z} / N \mathbb{Z}$ (ie, $\mathcal{L}^{\prime}$ has the same objects as $\mathcal{L}$ ). Then $\mathcal{L}$ is the localization of $\mathcal{L}^{\prime}$ with respect to the class of weak equivalences. This immediately follows from the fact that the class of weak equivalences in $\left(\mathcal{M}^{\mathcal{C}_{N}}\right)_{\text {cof }}$ admits a calculus of left fractions [18].

The following is one of the main technical results of the paper.

Proposition 3.3.5 Let $\mathcal{K}$ be the full subcategory of $\mathcal{L}$ consisting of all the objects $X$ with

proj.dim $Z^{(i)}(X)<N-1, \quad$ proj.dim $B^{(i)}(X)<N-1$,

for any $i \in \mathbb{Z} / N \mathbb{Z}$ (see 2.1.2). Then the functor

$$
\left.\mathcal{Q}\right|_{\mathcal{K}}: \mathcal{K} \longrightarrow \operatorname{Mod}-\pi_{*} P
$$

is full and faithful. 
Before starting to prove this proposition, we reformulate it in an equivalent way in terms of exact sequences. Consider $X, \tilde{X} \in \mathcal{K}$. Let $K$ denote the abelian group

$$
\operatorname{Hom}_{\mathrm{Ho}\left(\mathcal{M}^{\left.\mathcal{C}_{N}\right)}\right.}(X, \tilde{X})=[X, \tilde{X}],
$$

and $L$ denote the abelian group of commutative diagrams of the form

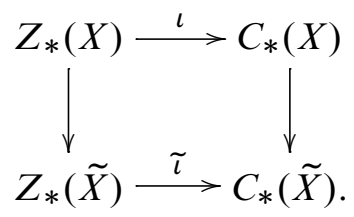

In other words,

$$
L=\operatorname{Hom}_{\left(\operatorname{Grmod}-\pi_{*} P\right)}\left(Z_{*}(X) \stackrel{\iota}{\rightarrow} C_{*}(X), Z_{*}(\tilde{X}) \stackrel{\tau}{\rightarrow} C_{*}(\tilde{X})\right),
$$

where $\underline{1}$ denotes the category $\bullet \longrightarrow \bullet$. To prove Proposition 3.3.5 it suffices to check that the morphism

$$
q: K \longrightarrow L
$$

induced by the functor $\mathcal{Q}$ is injective and its image consists of all those morphisms which respect the differentials, ie, which are morphisms of DG modules. Thanks to (3.3.2) any $f \in L$ induces a morphism on the cokernels

$$
B_{*}(X) \stackrel{\bar{f}}{\rightarrow} B_{*}(\tilde{X}) .
$$

By definition of $d: C_{*}(X) \longrightarrow C_{*}(X)[1]$, a morphism $f \in L$ is a DG morphism if and only if the outer square in the diagram

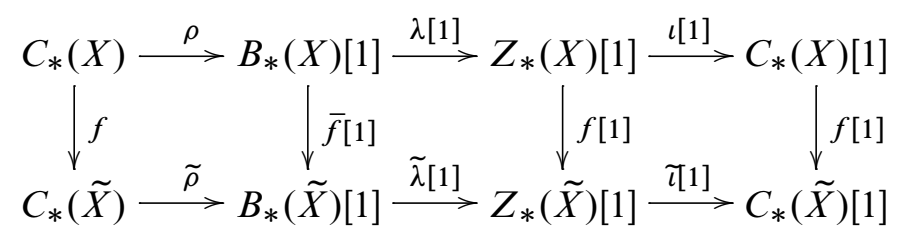

commutes. The left and right squares in the diagram are commutative. Therefore, since $\tilde{\imath}[1]: Z_{*}(\tilde{X})[1] \longrightarrow C_{*}(\tilde{X})[1]$ is a monomorphism and $\rho: C_{*}(X) \longrightarrow B_{*}(X)[1]$ an epimorphism, the outer square commutes if and only if the middle one does, ie, if and only if $f$ lies in the kernel of

$$
D: L \longrightarrow \operatorname{Hom}_{\pi_{*} P}\left(B_{*}(X), Z_{*}(\tilde{X})\right), \quad D(f)=\tilde{\lambda} \bar{f}-f \lambda .
$$

Thus, showing that the functor

$$
\left.\mathcal{Q}\right|_{\mathcal{K}}: \mathcal{K} \longrightarrow \operatorname{Mod}-\pi_{*} P
$$


is full and faithful is equivalent to showing that the sequence

$$
0 \longrightarrow K \stackrel{q}{\longrightarrow} L \stackrel{D}{\longrightarrow} \operatorname{Hom}_{\pi_{*} P}\left(B_{*}(X), Z_{*}(\tilde{X})\right)
$$

is exact, for any $X, \tilde{X} \in \mathcal{K}$. To prove the latter we describe $K$ and $L$ in terms of some exact sequences.

Let us start with $K$.

Lemma 3.3.7 For any $X, \tilde{X} \in \mathcal{K}$, there is a natural exact sequence

$$
\begin{aligned}
& 0 \longrightarrow \operatorname{Hom}_{\pi_{*} P}\left(B_{*}(X)[1], Z_{*}(\tilde{X})\right) \longrightarrow \longrightarrow \\
& \operatorname{Hom}_{\pi_{*} P}\left(B_{*}(X), B_{*}(\tilde{X})\right) \oplus \operatorname{Hom}_{\pi_{*} P}\left(Z_{*}(X), Z_{*}(\tilde{X})\right) \longrightarrow \\
& \operatorname{Ext}_{\pi_{*} P}^{1}\left(B_{*}(X)[1], Z_{*}(\tilde{X})\right) \oplus \operatorname{Hom}_{\pi_{*} P}\left(B_{*}(X), Z_{*}(\tilde{X})\right) .
\end{aligned}
$$

Proof By Remark 2.5.8, we have a pullback square of based simplicial sets

$$
\operatorname{Map}_{\mathcal{M}^{\mathcal{C}_{N}}}(X, \tilde{X}) \longrightarrow \prod_{i} \operatorname{Map}_{\mathcal{M}}\left(X_{\zeta_{i}}, \tilde{X}_{\zeta_{i}}\right)
$$

where $\phi$ and $\psi$ are induced by

$$
X_{\beta_{i-1}} \vee X_{\beta_{i}} \stackrel{\left(k_{i}, l_{i}\right)}{\longrightarrow} X_{\zeta_{i}}, \quad \tilde{X}_{\beta_{i}} \stackrel{\left(\tilde{k}_{i+1}, \tilde{l}_{i}\right)}{\longrightarrow} \tilde{X}_{\zeta_{i+1}} \times \tilde{X}_{\zeta_{i}}, \quad i \in \mathbb{Z} / N \mathbb{Z}
$$

respectively. Without loss of generality we may assume that $\tilde{X}$ is fibrant in $\mathcal{M}^{\mathcal{C}_{N}}$, ie, $\tilde{X}_{\beta_{i}}$ and $\tilde{X}_{\zeta_{i}}, i \in \mathbb{Z} / N \mathbb{Z}$ are fibrant in $\mathcal{M}$. Besides, since $X$ is a cofibrant object in $\mathcal{M}^{\mathcal{C}_{N}}$, we can conclude that

$$
X_{\beta_{i-1}} \vee X_{\beta_{i}} \stackrel{\left(k_{i}, l_{i}\right)}{\longrightarrow} X_{\zeta_{i}}
$$

is a cofibration and $X_{\beta_{i}}, X_{\zeta_{i}}$ are cofibrant, for any $i \in \mathbb{Z} / N \mathbb{Z}$ (see Example 2.5.5). This, by the axiom SM7 for simplicial model categories implies that all objects in (3.3.8) are fibrant and $\phi$ is a Kan fibration. Next, as $\mathcal{M}$ is stable, the simplicial sets and maps involved in (3.3.8) are infinite loop spaces and infinite loop maps, respectively. In particular, all the objects are homotopy associative and homotopy commutative $H$-spaces and all the morphisms are $H$-space maps. Thus, by Lemma 2.6.3, the diagram (3.3.8) gives a long exact Mayer-Vietoris sequence. Let us identify the terms of this long exact sequence. Since $X$ is cofibrant and $\tilde{X}$ is fibrant, the group 
$\pi_{0}\left(\operatorname{Map}_{\mathcal{M}^{c_{N}}}(X, \tilde{X})\right)$ is isomorphic to $[X, \tilde{X}]=K$, by [9, 2.3.10]. As noted above, $X_{\beta_{i}}$ and $X_{\zeta_{i}}, i \in \mathbb{Z} / N \mathbb{Z}$ are cofibrant and $\tilde{X}_{\beta_{i}}, \tilde{X}_{\zeta_{i}}$ are fibrant for all $i \in \mathbb{Z} / N \mathbb{Z}$. Hence, using again $[9,2.3 .10]$, we get isomorphisms

$$
\begin{aligned}
\pi_{n}\left(\operatorname{Map}_{\mathcal{M}}\left(X_{\beta_{i}}, \tilde{X}_{\beta_{i}}\right)\right) & \cong\left[\Sigma^{n} X_{\beta_{i}}, \tilde{X}_{\beta_{i}}\right], \\
\pi_{n}\left(\operatorname{Map}_{\mathcal{M}}\left(X_{\zeta_{i}}, \tilde{X}_{\zeta_{i}}\right)\right) & \cong\left[\Sigma^{n} X_{\zeta_{i}}, \tilde{X}_{\zeta_{i}}\right], \\
\pi_{n}\left(\operatorname{Map}_{\mathcal{M}}\left(X_{\beta_{i-1}}, \tilde{X}_{\zeta_{i}}\right)\right) & \cong\left[\Sigma^{n} X_{\beta_{i-1}}, \tilde{X}_{\zeta_{i}}\right], \\
\pi_{n}\left(\operatorname{Map}_{\mathcal{M}}\left(X_{\beta_{i}}, \tilde{X}_{\zeta_{i}}\right)\right) & \cong\left[\Sigma^{n} X_{\beta_{i}}, \tilde{X}_{\zeta_{i}}\right] .
\end{aligned}
$$

Thus, the final part of the Mayer-Vietoris sequence of the square (3.3.8) looks as follows:

$$
\begin{aligned}
& \bigoplus_{i}\left[\Sigma X_{\beta_{i}}, \tilde{X}_{\beta_{i}}\right] \oplus \bigoplus_{i}\left[\Sigma X_{\zeta_{i}}, \tilde{X}_{\zeta_{i}}\right] \longrightarrow \\
& \bigoplus_{i}\left[\Sigma X_{\beta_{i-1}}, \tilde{X}_{\zeta_{i}}\right] \oplus \bigoplus_{i}\left[\Sigma X_{\beta_{i}}, \tilde{X}_{\zeta_{i}}\right] \stackrel{\partial}{\longrightarrow} \\
& K \longrightarrow \bigoplus_{i}\left[X_{\beta_{i}}, \tilde{X}_{\beta_{i}}\right] \oplus \bigoplus_{i}\left[X_{\zeta_{i}}, \tilde{X}_{\zeta_{i}}\right] \longrightarrow \\
& \bigoplus_{i}\left[X_{\beta_{i-1}}, \tilde{X}_{\zeta_{i}}\right] \oplus \bigoplus_{i}\left[X_{\beta_{i}}, \tilde{X}_{\zeta_{i}}\right],
\end{aligned}
$$

where $\partial$ is the connecting homomorphism.

Next we need more explicit descriptions of the terms involved in this exact sequence. Proposition 3.2.1 yields the following isomorphisms:

$$
\begin{aligned}
{\left[X_{\beta_{i}}, \tilde{X}_{\beta_{i}}\right] } & \cong \operatorname{Hom}_{\pi_{*} P}\left(B^{(i)}(X), B^{(i)}(\tilde{X})\right), \\
{\left[X_{\zeta_{i}}, \tilde{X}_{\zeta_{i}}\right] } & \cong \operatorname{Hom}_{\pi_{*} P}\left(Z^{(i)}(X), Z^{(i)}(\tilde{X})\right), \\
{\left[X_{\beta_{i}}, \tilde{X}_{\zeta_{i}}\right] } & \cong \operatorname{Hom}_{\pi_{*} P}\left(B^{(i)}(X), Z^{(i)}(\tilde{X})\right), \\
{\left[\Sigma X_{\beta_{i-1}}, \tilde{X}_{\zeta_{i}}\right] } & \cong \operatorname{Hom}_{\pi_{*} P}\left(B^{(i-1)}(X)[1], Z^{(i)}(\tilde{X})\right), \\
{\left[X_{\beta_{i-1}}, \tilde{X}_{\zeta_{i}}\right] } & \cong \operatorname{Ext}_{\pi_{*} P}^{1}\left(B^{(i-1)}(X)[1], Z^{(i)}(\tilde{X})\right), \\
{\left[\Sigma X_{\beta_{i}}, \tilde{X}_{\beta_{i}}\right] } & \cong \operatorname{Ext}_{\pi_{*} P}^{N-1}\left(B^{(i)}(X)[N], B^{(i)}(\tilde{X})\right), \\
{\left[\Sigma X_{\zeta_{i}}, \tilde{X}_{\zeta_{i}}\right] } & \cong \operatorname{Ext}_{\pi_{*} P}^{N-1}\left(Z^{(i)}(X)[N], Z^{(i)}(\tilde{X})\right), \\
{\left[\Sigma X_{\beta_{i}}, \tilde{X}_{\zeta_{i}}\right] } & \cong \operatorname{Ext}_{\pi_{*} P}^{N-1}\left(B^{(i)}(X)[N], Z^{(i)}(\tilde{X})\right) .
\end{aligned}
$$

Since $X \in \mathcal{K}$, the last three Ext-groups vanish and therefore

$$
\left[\Sigma X_{\beta_{i}}, \tilde{X}_{\beta_{i}}\right]=0, \quad\left[\Sigma X_{\zeta_{i}}, \tilde{X}_{\zeta_{i}}\right]=0, \quad\left[\Sigma X_{\beta_{i}}, \tilde{X}_{\zeta_{i}}\right]=0 .
$$


Consequently, one obtains a natural exact sequence describing $K$, namely

$$
\begin{aligned}
0 \longrightarrow & \bigoplus_{i} \operatorname{Hom}_{\pi_{*} P}\left(B^{(i-1)}(X)[1], Z^{(i)}(\tilde{X})\right) \stackrel{\partial}{\longrightarrow} K \longrightarrow \\
& \bigoplus_{i} \operatorname{Hom}_{\pi_{*} P}\left(B^{(i)}(X), B^{(i)}(\tilde{X})\right) \oplus \bigoplus_{i} \operatorname{Hom}_{\pi_{*} P}\left(Z^{(i)}(X), Z^{(i)}(\tilde{X})\right) \longrightarrow \\
& \bigoplus_{i} \operatorname{Ext}_{\pi_{*} P}^{1}\left(B^{(i-1)}(X)[1], Z^{(i)}(\tilde{X})\right) \oplus \bigoplus_{i} \operatorname{Hom}_{\pi_{*} P}\left(B^{(i)}(X), Z^{(i)}(\tilde{X})\right) .
\end{aligned}
$$

As $B^{(i)}(X), B^{(i)}(\tilde{X}), Z^{(i)}(X), Z^{(i)}(\tilde{X}) \in \mathcal{B}[i], i \in \mathbb{Z} / N \mathbb{Z}$, we may rewrite this exact sequence as

$$
\begin{aligned}
0 \longrightarrow \operatorname{Hom}_{\pi_{*} P}\left(B_{*}(X)[1], Z_{*}(\tilde{X})\right) \stackrel{\partial}{\longrightarrow} K \longrightarrow \\
\operatorname{Hom}_{\pi_{*} P}\left(B_{*}(X), B_{*}(\tilde{X})\right) \oplus \operatorname{Hom}_{\pi_{*} P}\left(Z_{*}(X), Z_{*}(\tilde{X})\right) \longrightarrow \\
\operatorname{Ext}_{\pi_{*} P}^{1}\left(B_{*}(X)[1], Z_{*}(\tilde{X})\right) \oplus \operatorname{Hom}_{\pi_{*} P}\left(B_{*}(X), Z_{*}(\tilde{X})\right) .
\end{aligned}
$$

Let us now describe $L=\operatorname{Hom}_{\left(\operatorname{Grmod}-\pi_{*} P\right) \underline{1}}\left(Z_{*}(X) \stackrel{\iota}{\rightarrow} C_{*}(X), Z_{*}(\tilde{X}) \stackrel{\widetilde{\iota}}{\rightarrow} C_{*}(\tilde{X})\right)$.

Lemma 3.3.9 For any $X, \tilde{X} \in \mathcal{K}$, there is a natural exact sequence

$$
\begin{aligned}
0 \longrightarrow \operatorname{Hom}_{\pi_{*} P}\left(B_{*}(X)[1], Z_{*}(\tilde{X})\right) \longrightarrow L \longrightarrow & \longrightarrow \\
\operatorname{Hom}_{\pi_{*} P}\left(B_{*}(X), B_{*}(\tilde{X})\right) \oplus \operatorname{Hom}_{\pi_{*} P}\left(Z_{*}(X), Z_{*}(\tilde{X})\right) \longrightarrow & \longrightarrow \operatorname{Ext}_{\pi_{*} P}^{1}\left(B_{*}(X)[1], Z_{*}(\tilde{X})\right) .
\end{aligned}
$$

Proof First note that any $f \in L$ yields (as we have already mentioned) a diagram

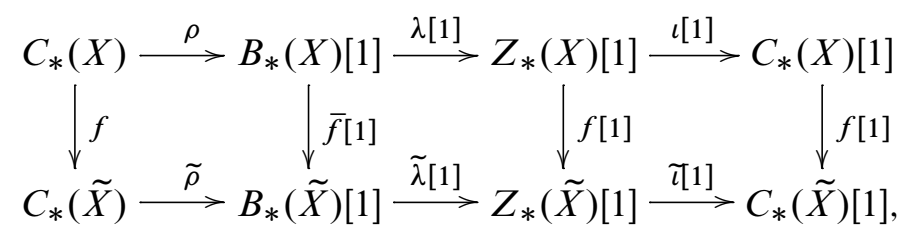

in which the left and the right squares commute. Consider the map $\sigma: L \longrightarrow \operatorname{Hom}_{\pi_{*} P}\left(B_{*}(X), B_{*}(\tilde{X})\right) \oplus \operatorname{Hom}_{\pi_{*} P}\left(Z_{*}(X), Z_{*}(\tilde{X})\right), \quad \sigma(f)=(\bar{f}, f)$. 
The kernel of $\sigma$ consists of morphisms of the form $(0, G) \in L$ such that the diagram

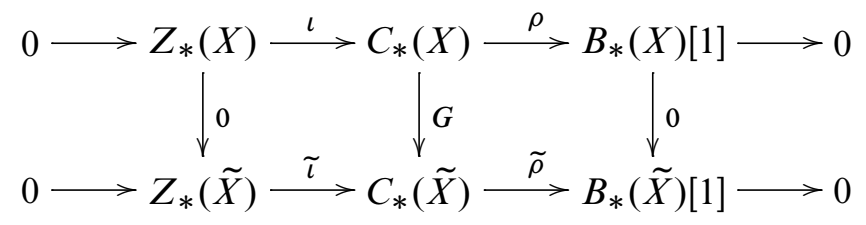

commutes, ie, we can identify the kernel with the subgroup of $\operatorname{Hom}_{\pi_{*} P}\left(C_{*}(X), C_{*}(\tilde{X})\right)$ consisting of those $G: C_{*}(X) \longrightarrow C_{*}(\tilde{X})$ for which $G \iota=0$ and $\tilde{\rho} G=0$. It then follows from the Snake Lemma that $G \in \operatorname{Ker} \sigma$ if and only if $G$ can be factored as

$$
C_{*}(X) \stackrel{\rho}{\longrightarrow} B_{*}(X)[1] \stackrel{g}{\longrightarrow} Z_{*}(\tilde{X}) \stackrel{\tilde{\iota}}{\longrightarrow} C_{*}(\tilde{X})
$$

for some $g$. Since $\rho$ is an epimorphism and $\tilde{\iota}$ a monomorphism, $\operatorname{Ker} \sigma$ is isomorphic to

$$
\operatorname{Hom}_{\pi_{*} P}\left(B_{*}(X)[1], Z_{*}(\tilde{X})\right)
$$

and we get an exact sequence

$$
\begin{aligned}
0 \longrightarrow & \operatorname{Hom}_{\pi_{*} P}\left(B_{*}(X)[1], Z_{*}(\tilde{X})\right) \stackrel{b}{\longrightarrow} L \stackrel{\sigma}{\longrightarrow} \\
& \operatorname{Hom}_{\pi_{*} P}\left(B_{*}(X), B_{*}(\tilde{X})\right) \oplus \operatorname{Hom}_{\pi_{*} P}\left(Z_{*}(X), Z_{*}(\tilde{X})\right),
\end{aligned}
$$

where

$$
b(g)=(0, \tilde{\imath} g \rho) .
$$

Then, in order to give a more precise description of $L$, we would like to examine $\operatorname{Im} \sigma$. For this purpose, observe that

$$
\begin{aligned}
& S: 0 \longrightarrow Z_{*}(X) \stackrel{\iota}{\longrightarrow} C_{*}(X) \stackrel{\rho}{\longrightarrow} B_{*}(X)[1] \longrightarrow 0, \\
& \tilde{S}: 0 \longrightarrow Z_{*}(\tilde{X}) \stackrel{\tilde{\iota}}{\longrightarrow} C_{*}(\tilde{X}) \stackrel{\tilde{\rho}}{\longrightarrow} B_{*}(\tilde{X})[1] \longrightarrow 0,
\end{aligned}
$$

represent elements of $\operatorname{Ext}_{\pi_{*} P}^{1}\left(B_{*}(X)[1], Z_{*}(X)\right)$ and $\operatorname{Ext}_{\pi_{*} P}^{1}\left(B_{*}(\tilde{X})[1], Z_{*}(\tilde{X})\right)$, respectively. Define a homomorphism

$\operatorname{Hom}_{\pi_{*} P}\left(B_{*}(X), B_{*}(\tilde{X})\right) \oplus \operatorname{Hom}_{\pi_{*} P}\left(Z_{*}(X), Z_{*}(\tilde{X})\right) \stackrel{v}{\longrightarrow} \operatorname{Ext}_{\pi_{*} P}^{1}\left(B_{*}(X)[1], Z_{*}(\tilde{X})\right)$,

$$
v(u, w)=u[1]^{*}(\widetilde{S})-w_{*}(S) .
$$


We claim that $\operatorname{Im} \sigma=\operatorname{Ker} v$. Indeed, $(u, w) \in \operatorname{Im} \sigma$ if and only if there exists $h: C_{*}(X) \longrightarrow C_{*}(\tilde{X})$ such that the diagram

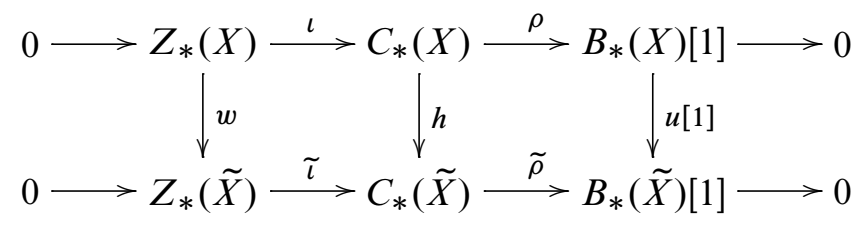

commutes. But the latter is the case if and only if $w_{*}(S)=u[1]^{*}(\widetilde{S})$, ie, $(u, w) \in \operatorname{Ker} v$. Hence, one concludes that there is an exact sequence

$0 \longrightarrow \operatorname{Hom}_{\pi_{*} P}\left(B_{*}(X)[1], Z_{*}(\tilde{X})\right) \stackrel{b}{\longrightarrow} L \stackrel{\sigma}{\longrightarrow}$

$$
\begin{array}{r}
\operatorname{Hom}_{\pi_{*} P}\left(B_{*}(X), B_{*}(\tilde{X})\right) \oplus \operatorname{Hom}_{\pi_{*} P}\left(Z_{*}(X), Z_{*}(\tilde{X})\right) \stackrel{v}{\longrightarrow} \\
\operatorname{Ext}_{\pi_{*} P}^{1}\left(B_{*}(X)[1], Z_{*}(\tilde{X})\right) .
\end{array}
$$

Proof of Proposition 3.3.5 As we already explained, proving Proposition 3.3.5 is equivalent to showing that the sequence (3.3.6) is exact. For this one has to compare the exact sequences describing $K$ and $L$ from the previous two lemmas. In other words, we have to check that the diagram

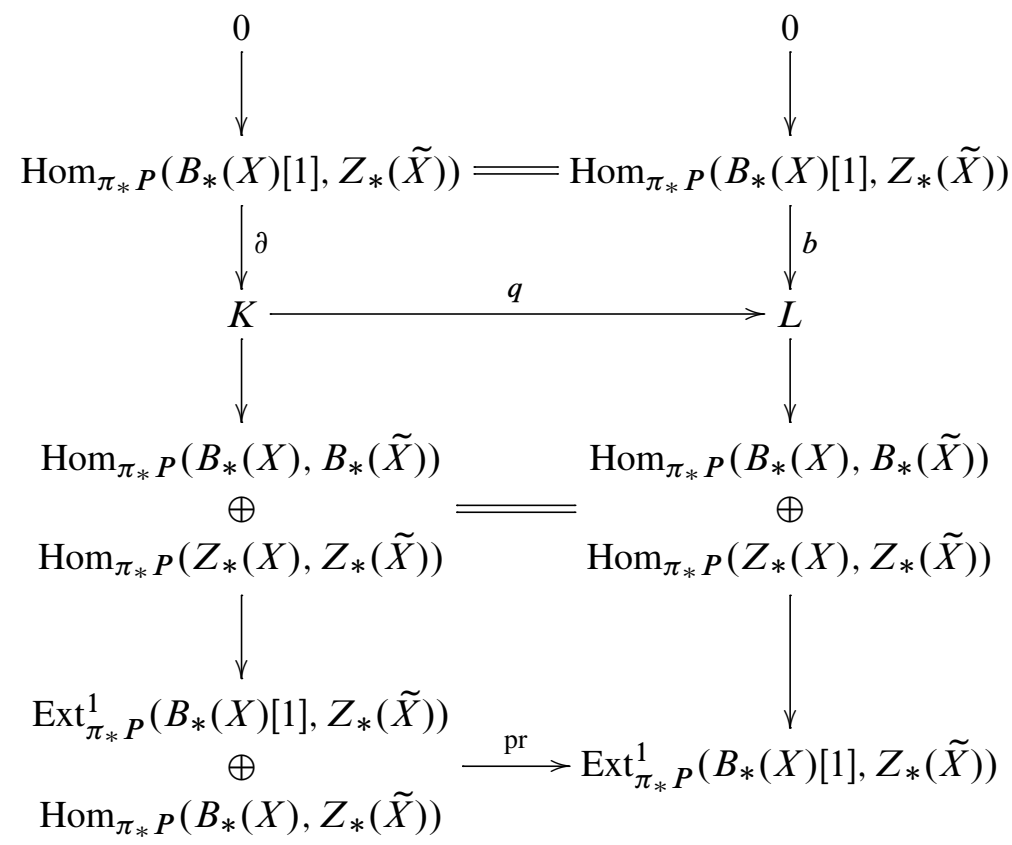


commutes. It is clear that the middle square in this diagram is commutative. The commutativity of the lower one is an immediate formal consequence of the construction of the Adams spectral sequence (Proposition 2.2.3). It thus remains to check that

$$
q \partial=b
$$

which needs a detailed verification.

Fix $j \in \mathbb{Z} / N \mathbb{Z}$ and take $\theta \in \operatorname{Hom}_{\pi_{*} P}\left(B^{(j-1)}(X)[1], Z^{(j)}(\tilde{X})\right)$. In order to compute $\partial(\theta)$, consider the isomorphisms

$$
\pi_{1}\left(\operatorname{Map}_{\mathcal{M}}\left(X_{\beta_{j-1}}, \tilde{X}_{\zeta_{j}}\right)\right) \cong\left[\Sigma X_{\beta_{j-1}}, \tilde{X}_{\zeta_{j}}\right] \cong \operatorname{Hom}_{\pi_{*} P}\left(B^{(j-1)}(X)[1], Z^{(j)}(\tilde{X})\right) .
$$

A representative of the homotopy class in $\left[\Sigma X_{\beta_{j-1}}, \tilde{X}_{\zeta_{j}}\right]$ to which $\theta$ corresponds will be denoted by

$$
\theta: S^{1} \wedge X_{\beta_{j-1}} \longrightarrow \tilde{X}_{\zeta_{j}}
$$

abusing notation. Let $\theta_{1}$ denote the composite

$$
\theta\left(\pi_{+} \wedge 1\right): I_{+} \wedge X_{\beta_{j-1}} \stackrel{\pi_{+} \wedge 1}{\longrightarrow} S^{1} \wedge X_{\beta_{j-1}} \stackrel{\theta}{\longrightarrow} \tilde{X}_{\zeta_{j}},
$$

where $\pi_{+}: I_{+} \rightarrow S^{1}$ is the projection. As noted above,

$$
X_{\beta_{j-1}} \vee X_{\beta_{j}} \stackrel{\left(k_{j}, l_{j}\right)}{\longrightarrow} X_{\zeta_{j}}
$$

is a cofibration. Besides, the map $\operatorname{in}_{1}: S^{0} \rightarrow I_{+}$is an acyclic cofibration of pointed simplicial sets (see 2.1.1). Therefore, the pushout product $[9,2.3 .11]$

$$
\operatorname{in}_{1} \square\left(k_{j}, l_{j}\right):\left(I_{+} \wedge\left(X_{\beta_{j-1}} \vee X_{\beta_{j}}\right)\right) \vee_{S^{0} \wedge\left(X_{\beta_{j-1}} \vee X_{\beta_{j}}\right)} S^{0} \wedge X_{\zeta_{j}} \longrightarrow I_{+} \wedge X_{\zeta_{j}}
$$

is an acyclic cofibration in $\mathcal{M}$. Then, since $\tilde{X}_{\zeta_{j}}$ is fibrant, there exists a morphism

$$
\Theta: I_{+} \wedge X_{\zeta_{j}} \longrightarrow \tilde{X}_{\zeta_{j}}
$$

making the diagram

$$
\begin{aligned}
& \left(I_{+} \wedge\left(X_{\beta_{j-1}} \vee X_{\beta_{j}}\right)\right) \vee_{S^{0} \wedge\left(X_{\beta_{j-1}} \vee X_{\beta_{j}}\right)} S^{0} \wedge X_{\zeta_{j}} \stackrel{\left(\theta_{1}, *, *\right)}{\longrightarrow} \tilde{X}_{\zeta_{j}} \\
& \operatorname{in}_{1} \square\left(k_{j}, l_{j}\right) \mid \\
& I_{+} \wedge X_{\zeta_{j}}
\end{aligned}
$$

commute. This morphism together with the map $\mathrm{in}_{0}: S^{0} \rightarrow I_{+}$(see 2.1.1) gives a composition $\theta_{0}=\Theta\left(\operatorname{in}_{0} \wedge 1\right): X_{\zeta_{j}} \cong S^{0} \wedge X_{\zeta_{j}} \longrightarrow \tilde{X}_{\xi_{j}}$. Clearly, one has $\theta_{0} k_{j}=*$ and $\theta_{0} l_{j}=*$. Consequently, there is $\hat{\theta} \in \operatorname{Hom}_{\mathcal{M}^{\mathcal{C}} N}(X, \tilde{X}) \cong\left(\operatorname{Map}_{\mathcal{M}^{\mathcal{C}_{N}}}(X, \tilde{X})\right)_{0}$ having 
$\theta_{0}$ as the $\zeta_{j}$-th component and zero as all the other components. By adjunction, the commutative diagram (3.3.11) is equivalent to the commutative square

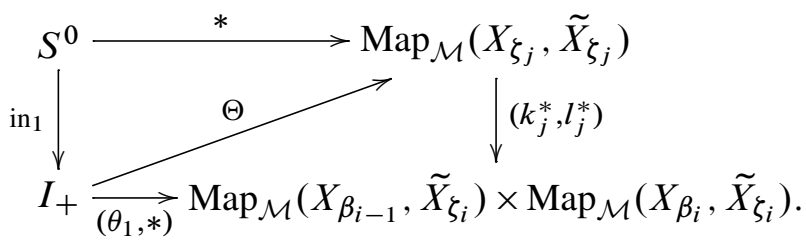

Therefore, by construction of $\partial$ (see Lemma 2.6.2), we conclude

$$
\partial(\theta)=[\hat{\theta}] \in \pi_{0}\left(\operatorname{Map}_{\mathcal{M}^{\mathcal{C}_{N}}}(X, \tilde{X})\right)=[X, \tilde{X}] .
$$

Thus one gets the desired computation of $\partial(\theta)$.

Next we show that

$$
q \partial(\theta)=b(\theta)
$$

ie,

$$
q([\hat{\theta}])=b(\theta) .
$$

For this purpose, we explicitly identify both sides of this equation and then compare them. In order to do so, note once again that the cone construction of Definition 2.4.3 is functorial on $\left(\mathcal{M}_{\text {cof }}\right)^{1}$ and hence we have a commutative diagram

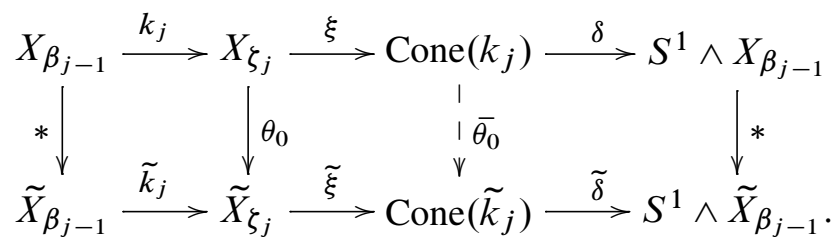

It follows from the universal property of pushout that there exists $\eta$ : $\operatorname{Cone}\left(k_{j}\right) \longrightarrow \tilde{X}_{\zeta_{j}}$ making the diagram

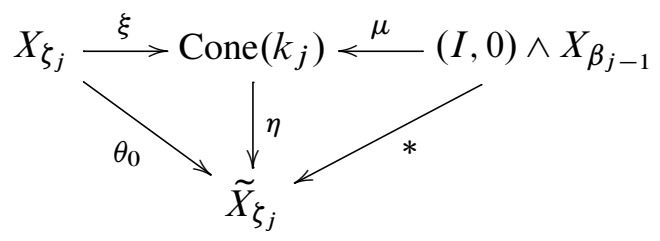

commute (since $\theta_{0} k_{j}=*$ ) and that $\overline{\theta_{0}}=\widetilde{\xi} \eta$. Then $\left(\theta_{0}\right.$ is null-homotopic via $\left.\Theta\right)$ and

$$
\begin{gathered}
q([\hat{\theta}])=\left(0, \pi_{*} \overline{\theta_{0}}\right)=\left(0, \pi_{*}(\tilde{\xi}) \pi_{*}(\eta)\right), \\
b(\theta)=\left(0, \pi_{*}(\tilde{\xi}) \pi_{*}(\theta) \pi_{*}(\delta)\right),
\end{gathered}
$$


by definitions of $q$ and $b$, respectively. Thus, to verify $q([\hat{\theta}])=b(\theta)$, it suffices to prove $\eta$ is homotopic to $\theta \delta$. This is done explicitly. Indeed, recall the simplicial set $I \times I$ is generated by the 2 -simplices $h_{0}=((0,0,1),(0,1,1))$ and $h_{1}=((0,1,1),(0,0,1))$ satisfying the relation $\partial_{1} h_{0}=\partial_{1} h_{1}$. Therefore, there is $\widehat{F}: I \times I \longrightarrow \operatorname{Map}_{\mathcal{M}}\left(X_{\beta_{j-1}}, \tilde{X}_{\zeta_{j}}\right)$ with $\widehat{F}\left(h_{0}\right)=\hat{F}\left(h_{1}\right)=s_{0} \theta_{1}\left(\theta_{1}\right.$ is considered as an element of $\left(\operatorname{Map}_{\mathcal{M}}\left(X_{\beta_{j-1}}, X_{\zeta_{j}}\right)\right)_{1}$; see Remark 2.3.3). It is easily seen that $\left.\widehat{F}\right|_{I \times 0}=*,\left.\widehat{F}\right|_{0 \times I}=*,\left.\hat{F}\right|_{I \times 1}=\theta_{1}$ and $\left.\widehat{F}\right|_{1 \times I}=\theta_{1}$; see 2.1.1. Hence, $\widehat{F}$ induces a simplicial map

$$
I_{+} \wedge(I, 0) \stackrel{\widetilde{F}}{\longrightarrow} \operatorname{Map}_{\mathcal{M}}\left(X_{\beta_{j-1}}, \tilde{X}_{\zeta_{j}}\right)
$$

with $\left.\tilde{F}\right|_{0 \wedge(I, 0)}=*,\left.\tilde{F}\right|_{1 \wedge(I, 0)}=\theta_{1}$, and $\left.\widetilde{F}\right|_{I_{+} \wedge 1}=\theta_{1}$ (see 2.1.1). Let

$$
I_{+} \wedge\left((I, 0) \wedge X_{\beta_{j-1}}\right) \stackrel{F}{\longrightarrow} \tilde{X}_{\zeta_{j}}
$$

be the adjoint of $\tilde{F}$. Then, $\left.F\right|_{0 \wedge(I, 0) \wedge X_{\beta_{j-1}}}=*,\left.F\right|_{1 \wedge(I, 0) \wedge X_{\beta_{j-1}}}=\theta(\pi \wedge 1)=\theta \delta \mu$ ( $\pi: I \rightarrow S^{1}$ is the projection) and $\left.F\right|_{I_{+} \wedge 1 \wedge X_{\beta_{j-1}}}=\theta_{1}$. The latter shows the diagram

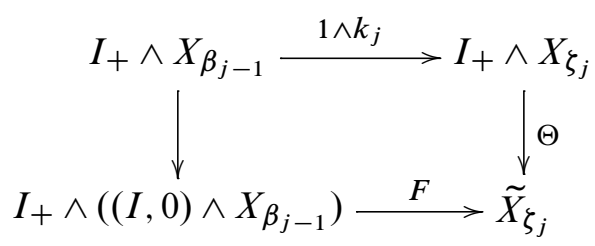

commutes. (Note that the left vertical arrow in this diagram comes from the morphism

$$
\left.X_{\beta_{j-1}} \cong S^{0} \wedge X_{\beta_{j-1}} \stackrel{\text { incl } \wedge 1}{\longrightarrow}(I, 0) \wedge X_{\beta_{j-1}} \cdot\right)
$$

On the other hand, by Definition 2.4.3, we have a pushout square

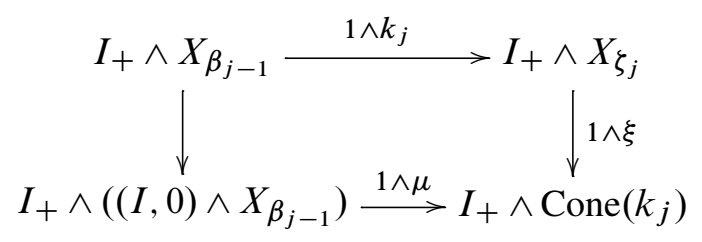

since $I_{+} \wedge-$ is left adjoint. Thus, there is $H: I_{+} \wedge \operatorname{Cone}\left(k_{j}\right) \longrightarrow \tilde{X}_{\zeta_{j}}$ so the diagram

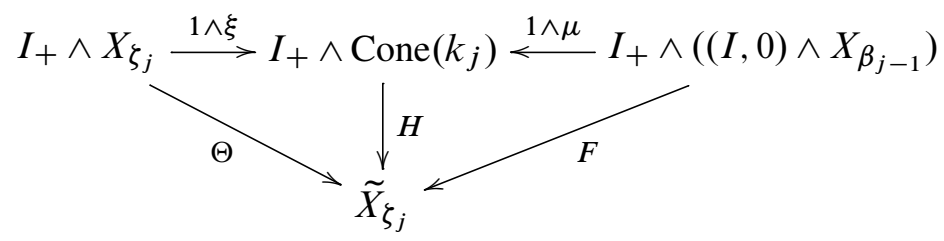


commutes. A trivial computation shows that $H$ is a left homotopy from $\eta$ to $\theta \delta$. Hence, $q \partial=b$.

Thus (3.3.10) commutes. Now a diagram chase shows that (3.3.6) is exact, and this completes the proof of Proposition 3.3.5.

Next we examine the image of $\left.\mathcal{Q}\right|_{\mathcal{K}}: \mathcal{K} \longrightarrow \operatorname{Mod}-\pi_{*} P$. By the construction of $\mathcal{Q}$ and the definition of $\mathcal{K}$, we have

proj.dim $\left(Z_{*}(X)=\operatorname{Ker} d\right)<N-1, \quad \operatorname{proj.dim}\left(B_{*}(X)=\operatorname{Im} d\right)<N-1, \quad X \in \mathcal{K}$.

These key properties completely determine the essential image of $\left.\mathcal{Q}\right|_{\mathcal{K}}$. Indeed, one has the following proposition.

Proposition 3.3.12 A differential graded $\pi_{*} P$-module $\left(C_{*}, d\right)$ is in the essential image of $\left.\mathcal{Q}\right|_{\mathcal{K}}$ if and only if

$$
\text { proj.dim } Z_{*}<N-1 \quad \text { proj.dim } B_{*}<N-1 \text {, }
$$

where $Z_{*}=\operatorname{Ker} d$ and $B_{*}=\operatorname{Im} d$.

Proof We have just mentioned that the "only if" part of this proposition is valid. Let us check that the "if" part holds as well.

Since $\pi_{*} P$ is $N$-sparse, we have the decompositions (see Subsection 3.2)

$$
\begin{aligned}
Z_{*} & =Z^{(0)} \oplus Z^{(1)} \oplus \cdots \oplus Z^{(N-1)}, \\
B_{*} & =B^{(0)} \oplus B^{(1)} \oplus \cdots \oplus B^{(N-1)}, \\
C_{*} & =C^{(0)} \oplus C^{(1)} \oplus \cdots \oplus C^{(N-1)},
\end{aligned}
$$

where $C^{(i)}, B^{(i)}, Z^{(i)} \in \mathcal{B}[i] i \in \mathbb{Z} / N \mathbb{Z}$. By Proposition 3.2.1(i) there are $X_{\zeta_{i}}, X_{\beta_{i}} \in \mathcal{E}_{i}$ such that

$$
Z^{(i)} \cong \pi_{*} X_{\zeta_{i}}, \quad B^{(i)} \cong \pi_{*} X_{\beta_{i}}
$$

for all $i \in \mathbb{Z} / N \mathbb{Z}$. We fix these isomorphisms once and for all. Without loss of generality one may assume that $X_{\beta_{i}}$ is cofibrant and $X_{\zeta_{i}}$ is bifibrant (ie, both cofibrant and fibrant) in $\mathcal{M}, i \in \mathbb{Z} / n \mathbb{Z}$. It then follows from Proposition 3.2.1(i) that there exist morphisms $l_{i}: X_{\beta_{i}} \longrightarrow X_{\zeta_{i}}$ in $\mathcal{M}$ such that the diagrams

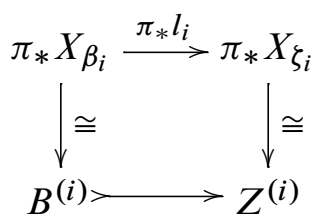


commute. Further, for any $i \in \mathbb{Z} / N \mathbb{Z}$, consider the extension

$$
E_{i}: 0 \longrightarrow Z^{(i)} \longrightarrow C^{(i)} \longrightarrow B^{(i-1)}[1] \longrightarrow 0 .
$$

By Proposition 3.2.1(ii), the extension class of $E_{i}$ corresponds to some element in $\left[X_{\beta_{i-1}}, X_{\zeta_{i}}\right]$ which is the homotopy class of some morphism $k_{i}: X_{\beta_{i-1}} \longrightarrow X_{\zeta_{i}}$ in $\mathcal{M}$. Consequently, we get a diagram

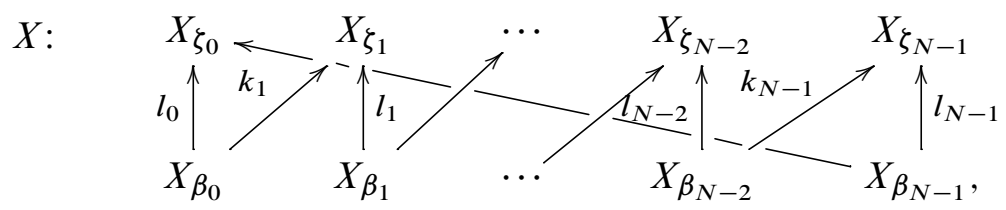

ie, an object in $\mathcal{M}^{\mathcal{C}_{N}}$. Without loss of generality we may assume that $X \in\left(\mathcal{M}^{\mathcal{C}_{N}}\right)_{\text {cof }}$ (otherwise we could replace $X$ cofibrantly). Besides, proj.dim $Z_{*}$ and proj.dim $B_{*}$ are less than $N-1$. Therefore, $X$ is an object of $\mathcal{K}$. Finally, the construction of $\mathcal{Q}$ and Remark 3.2.2 immediately imply that $\mathcal{Q}(X)$ is isomorphic to $C_{*}$.

In view of Proposition 3.3.5, one gets the following corollary.

Corollary 3.3.13 Let $\mathcal{M}$ be a stable model category and $P$ a compact generator of $\operatorname{Ho}(\mathcal{M})$. Suppose that the graded ring $\pi_{*} P$ is $N$-sparse and $\operatorname{gl.dim} \pi_{*} P<N-1$. Then the functor

$$
\mathcal{Q}: \mathcal{L} \longrightarrow \operatorname{Mod}-\pi_{*} P
$$

is an equivalence of categories (see Notation 3.3.1).

We conclude this subsection with the following proposition.

Proposition 3.3.14 For any $X \in \mathcal{L}$, there is a natural isomorphism of graded $\pi_{*} P-$ modules

$$
H_{*}(\mathcal{Q}(X)) \cong \pi_{*}(\operatorname{Hocolim} X)
$$

Proof As noted in Remark 3.3.4, $\mathcal{L}$ is the localization of $\mathcal{L}^{\prime} \subseteq\left(\mathcal{M}^{\mathcal{C}_{N}}\right)_{\text {cof }}$ at the class of weak equivalences. Let

$$
\mathcal{Q}^{\prime}=\mathcal{Q} \gamma: \mathcal{L}^{\prime} \longrightarrow \operatorname{Mod}-\pi_{*} P,
$$

where $\gamma: \mathcal{L}^{\prime} \longrightarrow \mathcal{L}$ is the localization functor (which is the identity on objects!). Clearly, to prove the proposition, it suffices to construct a natural isomorphism

$$
H_{*}\left(\mathcal{Q}^{\prime}(X)\right) \cong \pi_{*}(\operatorname{colim} X), \quad X \in \mathcal{L}^{\prime} .
$$


Recall that $X_{\beta_{i}}$ and $X_{\zeta_{i}}$ are cofibrant and

$$
X_{\beta_{i-1}} \vee X_{\beta_{i}} \stackrel{\left(k_{i}, l_{i}\right)}{\longrightarrow} X_{\zeta_{i}}
$$

is a cofibration for all $i \in \mathbb{Z} / N \mathbb{Z}$ (since $X$ is cofibrant in $\mathcal{M}^{\mathcal{C}_{N}}$ (see Example 2.5.5)). This implies that the coequalizer diagram

$$
\bigvee_{i} X_{\beta_{i}} \stackrel{k}{\underset{l}{\longrightarrow}} \bigvee_{i} X_{\zeta_{i}} \longrightarrow \operatorname{colim} X
$$

where $k=\bigvee_{i} k_{i}$ and $l=\bigvee_{i} l_{i}$, is in fact a homotopy coequalizer. Hence, we get a long exact sequence (see Corollary 2.6.5)

$$
\begin{aligned}
& \cdots \rightarrow \pi_{*}(\operatorname{colim} X)[-1] \stackrel{\partial}{\rightarrow} \bigoplus_{i} \pi_{*} X_{\beta_{i}} \stackrel{\pi_{*} l-\pi_{*} k}{\rightarrow} \bigoplus_{i} \pi_{*} X_{\zeta_{i}} \rightarrow \\
& \pi_{*}(\operatorname{colim} X) \stackrel{\partial}{\rightarrow} \bigoplus_{i} \pi_{*} X_{\beta_{i}}[1] \rightarrow \cdots .
\end{aligned}
$$

Since $X \in \mathcal{L}^{\prime}, \pi_{*} l=\bigoplus_{i} \pi_{*} l_{i}$ is a monomorphism and $\pi_{*} k=\bigoplus_{i} \pi_{*} k_{i}$ is zero. Therefore, the connecting homomorphisms of this long exact sequence vanish and we get a short exact sequence

$$
0 \longrightarrow \bigoplus_{i} \pi_{*} X_{\beta_{i}} \stackrel{\oplus_{i} \pi_{*} l_{i}}{\longrightarrow} \bigoplus_{i} \pi_{*} X_{\zeta_{i}} \longrightarrow \pi_{*}(\operatorname{colim} X) \longrightarrow 0,
$$

ie,

$$
0 \longrightarrow B_{*}(X) \stackrel{\lambda}{\longrightarrow} Z_{*}(X) \longrightarrow \pi_{*}(\operatorname{colim} X) \longrightarrow 0 .
$$

This short exact sequence shows that there is a natural isomorphism

$$
Z_{*}(X) / B_{*}(X) \cong \pi_{*}(\operatorname{colim} X) .
$$

The short exact sequence (3.3.2) together with the definition of the differential of $C_{*}(X)$ gives

$$
H_{*}\left(\mathcal{Q}^{\prime}(X)\right)=H_{*}\left(C_{*}(X)\right) \cong Z_{*}(X) / B_{*}(X) .
$$

Thus, we get a natural isomorphism

$$
H_{*}\left(\mathcal{Q}^{\prime}(X)\right) \cong \pi_{*}(\operatorname{colim} X) .
$$

\subsection{Proof of Theorem 3.1.4(i)}

Our aim is to construct a functor

$$
\mathscr{R}: \mathcal{D}\left(\pi_{*} P\right) \longrightarrow \operatorname{Ho}(\mathcal{M})
$$


and a natural isomorphism $\pi_{*} \circ \Re \cong H_{*}$.

By our assumption, the graded global homological dimension of $\pi_{*} P$ is less than $N$. This together with Proposition 3.3.12 implies that the essential image $\mathcal{Q}(\mathcal{K})$ of $\left.\mathcal{Q}\right|_{\mathcal{K}}$ contains all differential graded $\pi_{*} P$-modules which have underlying projective graded $\pi_{*} P$-modules. By Hinich [11, 2.2.5], it in particular contains all cofibrant DG $\pi_{*} P-$ modules. Therefore, the derived category $\mathcal{D}\left(\pi_{*} P\right)$ is equivalent to the localization $\mathcal{Q}(\mathcal{K})\left[\mathcal{V}^{-1}\right]$, where $\mathcal{V}$ is the class of quasi-isomorphisms in $\mathcal{Q}(\mathcal{K})$. Thus, in order to construct $\mathscr{R}$, we need a functor

$$
\mathscr{R}^{\prime}: \mathcal{Q}(\mathcal{K}) \longrightarrow \operatorname{Ho}(\mathcal{M})
$$

which sends quasi-isomorphisms of DG modules to isomorphisms. By Proposition 3.3.5,

$$
\left.\mathcal{Q}\right|_{\mathcal{K}}: \mathcal{K} \longrightarrow \mathcal{Q}(\mathcal{K})
$$

is an equivalence of categories and so we can choose an inverse $\left(\left.\mathcal{Q}\right|_{\mathcal{K}}\right)^{-1}: \mathcal{Q}(\mathcal{K}) \longrightarrow \mathcal{K}$. Define $\mathscr{R}^{\prime}$ to be the composite

$$
\mathcal{Q}(\mathcal{K}) \stackrel{\left(\left.\mathcal{Q}\right|_{\mathcal{K}}\right)^{-1}}{\longrightarrow} \mathcal{K} \subseteq \operatorname{Ho}\left(\mathcal{M}^{\mathcal{C}_{N}}\right) \stackrel{\text { Hocolim }}{\longrightarrow} \mathrm{Ho}(\mathcal{M}) .
$$

Then, Proposition 3.3.14 immediately yields a natural isomorphism

$$
\pi_{*}\left(\mathscr{R}^{\prime}(M)\right) \cong H_{*} M, \quad M \in \mathcal{Q}(\mathcal{K}) .
$$

In other words, the diagram

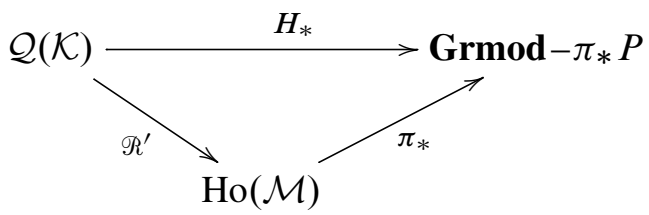

commutes up to a natural isomorphism. This shows that $\mathscr{R}^{\prime}$ sends quasi-isomorphisms to isomorphisms (since $\pi_{*}$ reflects isomorphisms). Consequently, $\mathscr{R}^{\prime}$ factors through the localization, ie, there exists

$$
\mathscr{R}: \mathcal{D}\left(\pi_{*} P\right) \longrightarrow \operatorname{Ho}(\mathcal{M})
$$

such that the diagram

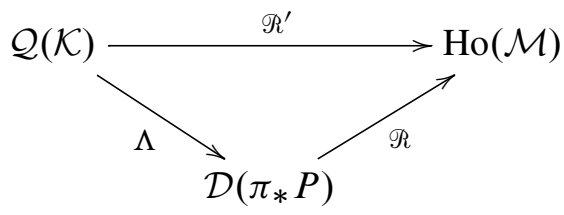


where $\Lambda$ is the localization functor, commutes up to a natural isomorphism. The universal property of localization and diagrams (3.4.1) and (3.4.2) give the desired result.

\subsection{Preservation of suspensions}

Proposition 3.5.1 Let $\mathcal{M}$ be a stable model category and $P$ a fixed compact generator of $\operatorname{Ho}(\mathcal{M})$. Suppose that the graded ring $\pi_{*} P$ is $N$-sparse and the (right) graded global homological dimension of $\pi_{*} P$ is less than $N$. Then the functor

$$
\mathscr{R}: \mathcal{D}\left(\pi_{*} P\right) \longrightarrow \operatorname{Ho}(\mathcal{M})
$$

commutes with suspensions.

Proof We consider the functor

$$
(-)^{\#}: \mathcal{L} \longrightarrow \mathcal{L}
$$

sending $X \in \mathcal{L}$ to $X^{\#}$ which is defined by

$$
\begin{gathered}
X_{\beta_{i}}^{\#}=S^{1} \wedge X_{\beta_{i-1}}, \quad X_{\zeta_{i}}^{\#}=S^{1} \wedge X_{\zeta_{i-1}}, \\
k_{i}^{\#}=1 \wedge k_{i-1}, \quad l_{i}^{\#}=1 \wedge l_{i-1}, \quad i \in \mathbb{Z} / N \mathbb{Z} .
\end{gathered}
$$

There is a natural isomorphism

$$
\operatorname{Hocolim}\left(X^{\#}\right) \cong \Sigma \text { Hocolim } X, \quad X \in \mathcal{L},
$$

in $\operatorname{Ho}(\mathcal{M})$. On the other hand, by construction of $\mathcal{Q}$, we have a natural isomorphism

$$
\mathcal{Q}\left(X^{\#}\right) \cong \mathcal{Q}(X)[1], \quad X \in \mathcal{L} ;
$$

see Remark 3.1.1. Consequently, for any $M \in \mathcal{Q}(\mathcal{K})$, there is a natural isomorphism

$$
\mathcal{Q}^{-1}(M[1]) \cong\left(\mathcal{Q}^{-1}(M)\right)^{\#} \text {. }
$$

By construction of $\mathscr{R}^{\prime}$, one gets

$$
\begin{aligned}
\mathscr{R}^{\prime}(M[1])=\operatorname{Hocolim}\left(\mathcal{Q}^{-1}(M[1])\right) & \cong \operatorname{Hocolim}\left(\left(\mathcal{Q}^{-1}(M)\right)^{\#}\right) \\
& \cong \Sigma \operatorname{Hocolim}\left(\mathcal{Q}^{-1}(M)\right)=\Sigma \mathscr{R}^{\prime}(M),
\end{aligned}
$$

whence we have a natural isomorphism

$$
\mathscr{R}(M[1]) \cong \Sigma \mathscr{R}(M), \quad M \in \mathcal{D}\left(\pi_{*} P\right)
$$




\section{Further properties of $\mathscr{R}$ and examples}

In this section we prove that the functor $\mathscr{R}$ need not be a derived functor of a Quillen functor. Besides, we give nontrivial examples where Theorem 3.1.4 can be applied.

First let us briefly recall derived mapping spaces.

\subsection{Derived mapping spaces}

For a pointed simplicial model category $\mathcal{M}$, one has the mapping space functor

$$
\operatorname{Map}_{\mathcal{M}}(-,-): \mathcal{M}^{\mathrm{op}} \times \mathcal{M} \longrightarrow \mathbf{s S e t}_{*} .
$$

The simplicial model category axioms imply that this functor is in fact a Quillen bifunctor (see [12, 4.2.1]). Therefore, we get the derived mapping space functor

$$
\operatorname{Map}_{\mathrm{Ho}(\mathcal{M})}(-,-): \operatorname{Ho}(\mathcal{M})^{\mathrm{op}} \times \operatorname{Ho}(\mathcal{M}) \longrightarrow \operatorname{Ho}\left(\mathbf{s S e t}_{*}\right) .
$$

If a model category $\mathcal{M}$ is not simplicial, then one does not have mapping spaces on the model level. However, one is still able to define derived mapping spaces. This is done in $[12,5.4]$ as follows.

Let $\mathcal{M}$ be a model category and $\mathcal{M}^{\Delta}$ denote the category of cosimplicial objects in $\mathcal{M}$. Consider the functor

$$
\mathrm{Ev}_{0}: \mathcal{M}^{\Delta} \longrightarrow \mathcal{M}
$$

which sends $X^{\bullet} \in \mathcal{M}^{\Delta}$ to the 0 -th space $X^{\bullet}[0]$. One can easily see that $\mathrm{Ev}_{0}$ has both a right and left adjoint. The right adjoint

$$
\mathbf{r}^{\bullet}: \mathcal{M} \longrightarrow \mathcal{M}^{\Delta}
$$

is the constant cosimplicial object functor. The left adjoint

$$
\mathbf{l}^{\bullet}: \mathcal{M} \longrightarrow \mathcal{M}^{\Delta}
$$

sends $X \in \mathcal{M}$ to the cosimplicial object whose $n$-th space is the $n+1$-fold coproduct of $X$ and whose structure morphisms are obtained from the coproduct inclusions and fold maps. The functors $\mathbf{I}^{\bullet}$ and $\mathbf{r}^{\bullet}$ come with a canonical natural transformation

$$
\mathbf{l}^{\bullet} \longrightarrow \mathbf{r}^{\bullet}
$$

which is the identity in degree 0 and the fold map in higher degrees.

Note there is a model structure on $\mathcal{M}^{\Delta}$, called the Reedy model structure (see [12, 5.2] for details), and that the adjunctions $\left(\mathbf{l}^{\bullet}, \mathrm{Ev}_{0}\right),\left(\mathrm{Ev}_{0}, \mathbf{r}^{\bullet}\right)$ are in fact Quillen adjunctions. 
Definition 4.1.1 $[12,5.2 .7]$ Suppose $\mathcal{M}$ is a model category and $X$ an object of $\mathcal{M}$. A cosimplicial frame on $X$ is a cosimplicial object $X^{\bullet} \in \mathcal{M}^{\Delta}$ together with a factorization

$$
\mathbf{l}^{\bullet} X \longrightarrow X^{\bullet} \longrightarrow \mathbf{r}^{\bullet} X
$$

of the canonical map

$$
\mathbf{l}^{\bullet} X \longrightarrow \mathbf{r}^{\bullet} X
$$

into a cofibration in $\mathcal{M}^{\Delta}$ followed by a weak equivalence (which is an isomorphism in degree zero).

The existence of such frames is shown in [12, 5.2.8].

Definition 4.1.2 Let $X, Y \in \operatorname{Ho}(\mathcal{M})$. The derived mapping space $\operatorname{Map}_{\mathrm{Ho}(\mathcal{M})}(X, Y)$ is defined by

$$
\operatorname{Map}_{\mathrm{Ho}(\mathcal{M})}(X, Y)=\operatorname{Hom}_{\mathcal{M}}\left(X^{\bullet}, Y^{f}\right) \in \mathbf{s S e t},
$$

where $X^{\bullet}$ is a cosimplicial frame of $X$ and $Y^{f}$ a fibrant replacement of $Y$.

It is proved in $[12,5.4]$ that the derived mapping space is a well-defined object of Ho(sSet), ie, it does not depend, up to homotopy, on the choice of the cosimplicial frame $X^{\bullet}$ and the fibrant replacement $Y^{f}$. Moreover, we have a functor

$$
\operatorname{Map}_{\mathrm{Ho}(\mathcal{M})}(-,-): \operatorname{Ho}(\mathcal{M})^{\mathrm{op}} \times \operatorname{Ho}(\mathcal{M}) \longrightarrow \operatorname{Ho}(\text { sSet }) .
$$

If $\mathcal{M}$ is pointed, then $\operatorname{Map}_{\mathrm{Ho}(\mathcal{M})}(X, Y)$ comes with a natural base point and it is a well-defined object of $\mathrm{Ho}\left(\mathbf{s S e t}_{*}\right)$.

Proposition 4.1.3 [12, 5.6.2] Suppose

$$
F: \mathcal{M} \rightleftarrows \mathcal{N}: G
$$

is a Quillen adjunction, where $F$ is a left adjoint. Then, for any $X \in \operatorname{Ho}(\mathcal{M})$ and $Y \in \operatorname{Ho}(\mathcal{N})$, there is a natural weak equivalence of derived mapping spaces

$$
\operatorname{Map}_{\mathrm{Ho}(\mathcal{N})}(\boldsymbol{L} F X, Y) \simeq \operatorname{Map}_{\mathrm{Ho}(\mathcal{M})}(X, \boldsymbol{R} G Y) .
$$

Remark 4.1.4 Let $A$ be a differential graded ring. The category Mod $-A$ with the model structure from Example 2.4.7 is not a simplicial model category. However, one can apply Definition 4.1.2 to Mod- $A$ and get derived mapping spaces. Note that since $\operatorname{Mod}-A$ is an additive category, $\operatorname{Map}_{\mathcal{D}(A)}(X, Y)$ is in fact a simplicial abelian group for any $X, Y \in \mathcal{D}(A)$. It is well known that every simplicial abelian group has the homotopy type of a product of Eilenberg-Mac Lane spaces (see eg, [9, III.2.18]). In particular, $\operatorname{Map}_{\mathcal{D}(A)}(X, Y)$ is homotopy equivalent to a product of Eilenberg-Mac Lane spaces for any $X, Y \in \mathcal{D}(A)$. 
Remark 4.1.5 There is an analog of the pushout product axiom for cosimplicial frames (see [12, 5.4.1 and 5.4.2]). Using this and the derived mapping spaces it is not difficult to see that the proofs given in Sections 3, 5 and 6 can be applied to stable model categories without a simplicial enrichment.

\subsection{The cases when $\mathscr{R}$ is not a derived functor}

The following shows that Proposition 4.1.3 is rather useful.

Theorem 4.2.1 Let $\mathcal{M}$ be a stable model category and $P$ a compact generator of $\operatorname{Ho}(\mathcal{M})$. Suppose that the graded ring $\pi_{*} P$ is $N$-sparse and gl.dim $\pi_{*} P<N$

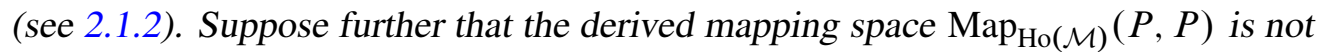
weakly equivalent to a product of Eilenberg-Mac Lane spaces. Then the functor

$$
\mathscr{R}: \mathcal{D}\left(\pi_{*} P\right) \longrightarrow \operatorname{Ho}(\mathcal{M})
$$

is not a derived functor of a Quillen functor.

Proof Consider the object

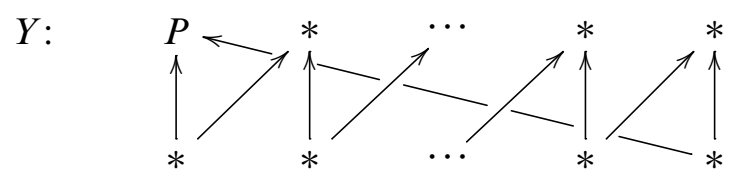

in $\mathcal{M}^{\mathcal{C}_{N}}$. Clearly, $Y \in \mathcal{K}$ (see Proposition 3.3.5). By construction of $\mathcal{Q}$,

$$
\mathcal{Q}(Y) \cong \pi_{*} P,
$$

where $\pi_{*} P$ is regarded as a DG $\pi_{*} P$-module with zero differential. On the other hand,

$$
\text { Hocolim } Y=\operatorname{colim} Y \cong P \text {. }
$$

Consequently,

$$
\mathscr{R}\left(\pi_{*} P\right) \cong P
$$

in $\operatorname{Ho}(\mathcal{M})$. Now assume that $\mathscr{R}$ is the derived functor of a left Quillen functor. Then $\mathscr{R}$ has a right adjoint

$$
\mathcal{G}: \operatorname{Ho}(\mathcal{M}) \longrightarrow \mathcal{D}\left(\pi_{*} P\right)
$$

and, in view of Proposition 4.1.3, we get a weak equivalence of derived mapping spaces

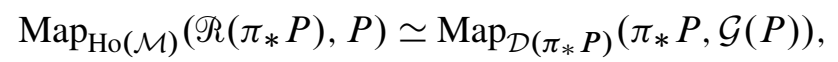

ie,

$$
\operatorname{Map}_{\mathrm{Ho}(\mathcal{M})}(P, P) \simeq \operatorname{Map}_{\mathcal{D}\left(\pi_{*} P\right)}\left(\pi_{*} P, \mathcal{G}(P)\right) .
$$


By Remark 4.1.4, the mapping space on the right is a product of Eilenberg-Mac Lane spaces, a contradiction.

One similarly shows that $\mathscr{R}$ cannot be the derived functor of a right Quillen functor.

Corollary 4.2.2 Let $R$ be a symmetric ring spectrum such that the graded homotopy ring $\pi_{*} R$ is $N$-sparse and $\mathbf{g l . d i m} \pi_{*} R<N$, and the infinite loop space $\Omega^{\infty} R$ is not weakly equivalent to a product of Eilenberg-Mac Lane spaces. Then the functor

$$
\mathscr{R}: \mathcal{D}\left(\pi_{*} R\right) \longrightarrow \mathcal{D}(R)
$$

is not derived from a Quillen functor.

Further, in view of Proposition 4.1.3 and Remark 4.1.4, one gets the following.

Proposition 4.2.3 Let $\mathcal{M}$ be a stable model category and $P$ a compact generator

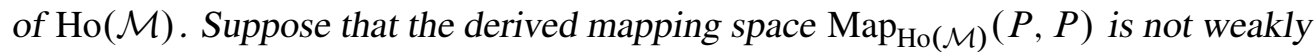
equivalent to a product of Eilenberg-Mac Lane spaces. Then there does not exist a zigzag of Quillen equivalences between the model categories $\mathcal{M}$ and $\mathbf{M o d}-\pi_{*} P$. In particular, if the graded ring $\pi_{*} P$ is $N$-sparse, gl.dim $\pi_{*} P<N$, and the functor

$$
\mathscr{R}: \mathcal{D}\left(\pi_{*} P\right) \longrightarrow \operatorname{Ho}(\mathcal{M})
$$

is an equivalence of categories (see Remark 6.3.1), then $\mathscr{R}$ cannot be derived from a zigzag of Quillen equivalences.

Corollary 4.2.4 Let $R$ be a symmetric ring spectrum. Suppose that the infinite loop space $\Omega^{\infty} R$ is not weakly equivalent to a product of Eilenberg-Mac Lane spaces. Then there does not exist a zigzag of Quillen equivalences between the model categories $\operatorname{Mod}-R$ and Mod $-\pi_{*} R$. In particular, if the graded homotopy ring $\pi_{*} R$ is $N$-sparse, gl.dim $\pi_{*} R<N$, and the functor

$$
\mathscr{R}: \mathcal{D}\left(\pi_{*} R\right) \longrightarrow \mathcal{D}(R)
$$

is an equivalence of categories, then $\mathscr{R}$ cannot be derived from a zigzag of Quillen equivalences.

\subsection{Examples}

Example 4.3.1 (Truncated Brown-Peterson spectra) Let $p$ be a prime, $n$ a natural number, and suppose that

$$
n+1<2(p-1) \text {. }
$$


Then we have that the truncated Brown-Peterson spectrum $B P\langle n\rangle$ satisfies the hypotheses of Theorem 1.1.1. Indeed, the homotopy ring of $B P\langle n\rangle$ looks as follows:

$$
\pi_{*} B P\langle n\rangle \cong \mathbb{Z}_{(p)}\left[v_{1}, \ldots, v_{n}\right], \quad\left|v_{i}\right|=2\left(p^{i}-1\right) .
$$

Clearly, gl.dim $\pi_{*} B P\langle n\rangle=n+1$ and $\pi_{*} B P\langle n\rangle$ is $2(p-1)$-sparse.

Note also that $\Omega^{\infty} B P\langle n\rangle$ is not weakly equivalent to a product of Eilenberg-Mac Lane spaces (see Corollary A.1.7). Therefore, in view of Corollary 4.2.2, the functor

$$
\mathscr{R}: \mathcal{D}\left(\mathbb{Z}_{(p)}\left[v_{1}, \ldots, v_{n}\right]\right) \longrightarrow \mathcal{D}(B P\langle n\rangle)
$$

is not a derived functor of a Quillen functor.

Example 4.3.2 (Johnson-Wilson spectra) The Johnson-Wilson spectrum $E(n)$ is obtained from $B P\langle n\rangle$ by inverting the generator $v_{n}$. In particular, for the homotopy ring of $E(n)$ one has

$$
\pi_{*} E(n) \cong \mathbb{Z}_{(p)}\left[v_{1}, \ldots, v_{n}, v_{n}{ }^{-1}\right], \quad\left|v_{i}\right|=2\left(p^{i}-1\right) .
$$

Note that $\operatorname{gl.dim} \pi_{*} E(n)=n$ and $\pi_{*} E(n)$ is $2(p-1)$-sparse. Therefore, if

$$
n<2(p-1),
$$

then Theorem 1.1.1 can be applied to $E(n)$. Furthermore, since $\Omega^{\infty} E(n)$ is not weakly equivalent to a product of Eilenberg-Mac Lane spaces (see Corollary A.1.8), the functor

$$
\mathscr{R}: \mathcal{D}\left(\mathbb{Z}_{(p)}\left[v_{1}, \ldots, v_{n}, v_{n}{ }^{-1}\right]\right) \longrightarrow \mathcal{D}(E(n))
$$

is not a derived functor of a Quillen functor.

\section{The one-dimensional case}

In this section we prove Theorem 3.1.4(ii).

\subsection{Technical lemmas}

The following well-known proposition immediately follows from the Adams spectral sequence of Proposition 2.2.3.

Proposition 5.1.1 (Universal coefficient theorem) Suppose $\mathcal{T}$ is a triangulated category with infinite coproducts, $P \in \mathcal{T}$ a compact generator, and let $\pi_{*}=\operatorname{Hom}_{\mathcal{T}}(P,-)_{*}$. 
Then for any $X$ with proj.dim $\pi_{*} X \leq 1$ (in Grmod $-\pi_{*} P$ ) and $Y \in \mathcal{T}$, there is a natural short exact sequence

$$
0 \longrightarrow \operatorname{Ext}_{\pi_{*} P}^{1}\left(\pi_{*} X[1], \pi_{*} Y\right) \longrightarrow \operatorname{Hom}_{\mathcal{T}}(X, Y) \stackrel{\pi_{*}}{\longrightarrow} \operatorname{Hom}_{\pi_{*} P}\left(\pi_{*} X, \pi_{*} Y\right) \longrightarrow 0 .
$$

In particular, $X$ is isomorphic to $Y$ in $\mathcal{T}$ if and only if $\pi_{*} X$ and $\pi_{*} Y$ are isomorphic as graded $\pi_{*} P$-modules.

Corollary 5.1.2 Let $\mathcal{T}, P$ and $\pi_{*}$ be as in Proposition 5.1.1, and suppose that $\pi_{*} P$ is $N$-sparse. Then any $X \in \mathcal{T}$ with proj.dim $\pi_{*} X \leq 1$ splits as follows:

$$
X \cong \bigoplus_{i \in \mathbb{Z} / N \mathbb{Z}} X^{(i)}, \quad \pi_{*} X^{(i)} \in \mathcal{B}[i],
$$

where $\mathcal{B}$ is as in Subsection 3.2.

Definition 5.1.3 Let $\mathcal{T}$ be a triangulated category. One says that a triangle

$$
X \stackrel{f}{\longrightarrow} Y \stackrel{g}{\longrightarrow} Z \stackrel{h}{\longrightarrow} \Sigma X
$$

in $\mathcal{T}$ is antidistinguished if the triangle

$$
X \stackrel{f}{\longrightarrow} Y \stackrel{g}{\longrightarrow} Z \stackrel{-h}{\longrightarrow} \Sigma X
$$

is distinguished.

Lemma 5.1.4 Let $\mathcal{M}$ be a (simplicial) stable model category, $G_{0}, G_{1}$ cofibrant objects of $\mathcal{M}, \omega: G_{0} \longrightarrow G_{1}$ a cofibration in $\mathcal{M}$, and $P$ a compact generator of $\operatorname{Ho}(\mathcal{M})$. Suppose that the graded ring $\pi_{*} P$ is $N$-sparse and $\operatorname{gl.dim} \pi_{*} P<N$. Further, assume that $\pi_{*} G_{0}, \pi_{*} G_{1}$ are in $\mathcal{B}[i]$ for fixed $i \in \mathbb{Z} / N \mathbb{Z}, \pi_{*} G_{0}, \pi_{*} G_{1}$ are projective graded $\pi_{*} P$-modules and $\pi_{*} \omega$ is a monomorphism. Then the functor

$$
\mathscr{R}: \mathcal{D}\left(\pi_{*} P\right) \longrightarrow \operatorname{Ho}(\mathcal{M})
$$

sends the antidistinguished triangle (see Remark 2.4.8)

$$
\pi_{*} G_{0} \stackrel{\pi_{*} \omega}{\longrightarrow} \pi_{*} G_{1} \longrightarrow C\left(\pi_{*} \omega\right) \stackrel{-\partial}{\longrightarrow} \pi_{*} G_{0}[1]
$$

to a triangle isomorphic to the elementary distinguished triangle

$$
G_{0} \stackrel{\omega}{\longrightarrow} G_{1} \longrightarrow \operatorname{Cone}(\omega) \longrightarrow S^{1} \wedge G_{0}
$$

of $\omega$ (see Definition 2.4.3). 
Proof By Proposition 3.3.12, since $\pi_{*} G_{0}, \pi_{*} G_{1}$ are projective, the triangle (5.1.5) is contained in $\mathcal{Q}(\mathcal{K})$. Let us construct a sequence

$$
A_{0} \stackrel{\alpha_{0}}{\longrightarrow} A_{1} \stackrel{\alpha_{1}}{\longrightarrow} A_{2} \stackrel{\alpha_{2}}{\longrightarrow} A_{3}
$$

in $\mathcal{K}$ which is mapped to the triangle (5.1.5) after applying $\mathcal{Q}$. Define

$A_{0}$ :

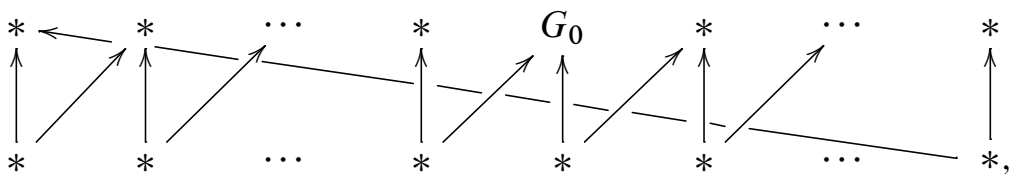

$A_{1}$ :

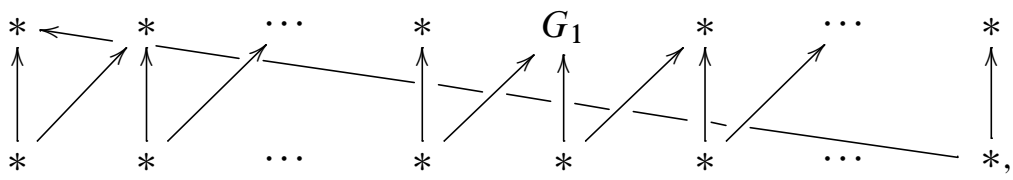

$A_{2}$ :

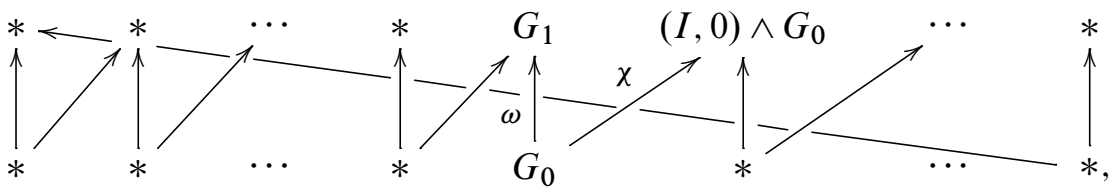

$A_{3}$ :

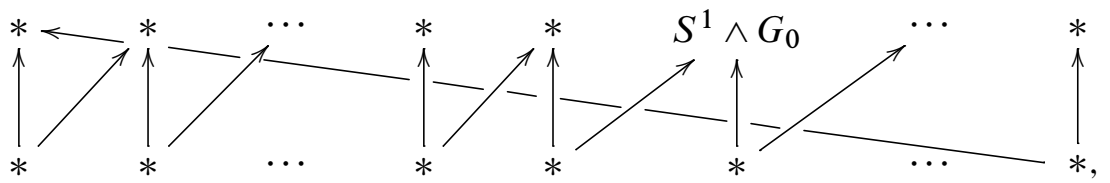

where $\chi: G_{0} \longrightarrow(I, 0) \wedge G_{0}$ is the canonical morphism and

$$
\begin{gathered}
\left(A_{0}\right)_{\zeta_{i}}=G_{0}, \\
\left(A_{1}\right)_{\zeta_{i}}=G_{1}, \\
\left(A_{2}\right)_{\zeta_{i}}=G_{1}, \quad\left(A_{2}\right)_{\zeta_{i+1}}=(I, 0) \wedge G_{0}, \quad\left(A_{2}\right)_{\beta_{i}}=G_{0}, \\
\left(A_{3}\right)_{\zeta_{i+1}}=S^{1} \wedge G_{0} .
\end{gathered}
$$

Next, define the nontrivial entries of $\alpha_{0}, \alpha_{1}$, and $\alpha_{2}$ by

$$
\left(\alpha_{0}\right)_{\zeta_{i}}=\omega, \quad\left(\alpha_{1}\right)_{\zeta_{i}}=1, \quad\left(\alpha_{2}\right)_{\zeta_{i+1}}=\pi \wedge 1,
$$

where $\pi: I \longrightarrow S^{1}$ is the projection. It follows from the construction of $\mathcal{Q}$ that the triangle

$$
\mathcal{Q}\left(A_{0}\right) \stackrel{\mathcal{Q}\left(\alpha_{0}\right)}{\longrightarrow} \mathcal{Q}\left(A_{1}\right) \stackrel{\mathcal{Q}\left(\alpha_{1}\right)}{\longrightarrow} \mathcal{Q}\left(A_{2}\right) \stackrel{\mathcal{Q}\left(\alpha_{2}\right)}{\longrightarrow} \mathcal{Q}\left(A_{3}\right) \cong \mathcal{Q}\left(A_{0}\right)[1]
$$


is isomorphic to (5.1.5). (Note that the morphism $\xi^{\prime}$ : Cone $(\chi) \longrightarrow S^{1} \wedge G_{0}$ induced from the arrow morphism

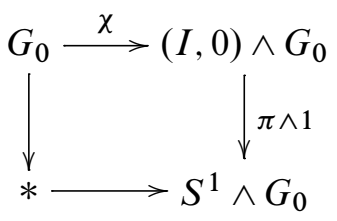

on the cones and the morphism $\xi$ : Cone $(\chi) \longrightarrow S^{1} \wedge G_{0}$ coming from the cone construction of Definition 2.4.3 differ by a sign in the homotopy category $\operatorname{Ho}(\mathcal{M})$. This explains the appearance of the minus sign before $\partial$ in (5.1.5).)

Applying the functor Hocolim to the sequence (5.1.6) gives the elementary distinguished triangle

$$
G_{0} \stackrel{\omega}{\longrightarrow} G_{1} \longrightarrow \text { Cone }(\omega) \longrightarrow S^{1} \wedge G_{0}
$$

since $A_{0}, A_{1}, A_{2}$ and $A_{3}$ are cofibrant. Now, by construction of $\mathscr{R}$, the desired result follows.

Lemma 5.1.7 Let $\mathcal{M}, P$ and $N$ be as in Theorem 3.1.4. Assume that the triangle

$$
F_{1} \stackrel{\iota}{\longrightarrow} F_{0} \longrightarrow M \longrightarrow F_{1}[1]
$$

is antidistinguished in $\mathcal{D}\left(\pi_{*} P\right)$, and $H_{*} F_{0}, H_{*} F_{1}$ are projective $\pi_{*} P$-modules, and $H_{*}(\iota)$ is a monomorphism. Then the triangle

$$
\mathscr{R}\left(F_{1}\right) \stackrel{\mathscr{R}(\iota)}{\longrightarrow} \mathscr{R}\left(F_{0}\right) \longrightarrow \mathscr{R}(M) \longrightarrow \mathscr{R}\left(F_{1}[1]\right) \cong \Sigma \mathscr{R}\left(F_{1}\right)
$$

is distinguished.

Proof By Corollary 5.1.2, we have splittings

$$
F_{0} \cong \bigoplus_{i \in \mathbb{Z} / N \mathbb{Z}} F_{0}^{(i)}, \quad F_{1} \cong \bigoplus_{i \in \mathbb{Z} / N \mathbb{Z}} F_{1}^{(i)}
$$

in $\mathcal{D}\left(\pi_{*} P\right)$, where $H_{*} F_{0}^{(i)}, H_{*} F_{1}^{(i)} \in \mathcal{B}[i]$. Since $H_{*} F_{1}$ is projective, it follows by Lemma 2.2.2(i) that for any $i, j \in \mathbb{Z} / N \mathbb{Z}$,

$$
\left[F_{1}^{(i)}, F_{0}^{(j)}\right] \cong \operatorname{Hom}_{\pi_{*} P}\left(H_{*} F_{1}^{(i)}, H_{*} F_{0}^{(j)}\right) .
$$

In particular,

$$
\left[F_{1}^{(i)}, F_{0}^{(j)}\right]=0, \quad i \neq j
$$


whence,

$$
\left[F_{1}, F_{0}\right] \cong \bigoplus_{i \in \mathbb{Z} / N \mathbb{Z}}\left[F_{1}^{(i)}, F_{0}^{(i)}\right]
$$

This implies that the morphism $\iota: F_{1} \longrightarrow F_{0}$ splits as well. More precisely, there are morphisms $\iota^{(i)}: F_{1}^{(i)} \longrightarrow F_{0}^{(i)}$ such that the diagram

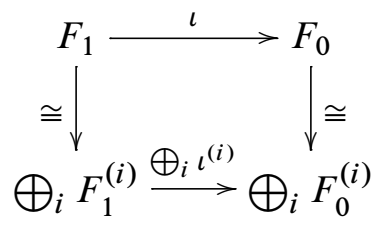

commutes in $\mathcal{D}\left(\pi_{*} P\right)$. Using this we see that the antidistinguished triangle

$$
F_{1} \stackrel{\iota}{\longrightarrow} F_{0} \longrightarrow M \longrightarrow F_{1}[1]
$$

is isomorphic to a finite sum of triangles of the form (5.1.5). As $\mathscr{R}$ is an additive functor, Lemma 5.1.4 completes the proof.

\subsection{Proof of Theorem 3.1.4(ii)}

First we show the essential surjectivity. Consider $X \in \operatorname{Ho}(\mathcal{M})$ with proj.dim $\pi_{*} X \leq 1$. Since

$$
H_{*}: \mathcal{D}\left(\pi_{*} P\right) \longrightarrow \mathbf{G r m o d}-\pi_{*} P
$$

is essentially surjective, there is $M \in \mathcal{D}\left(\pi_{*} P\right)$ such that $H_{*} M$ and $\pi_{*} X$ are isomorphic as graded $\pi_{*} P$-modules. By Theorem 3.1.4(i), $\pi_{*} \mathscr{R} \cong H_{*}$ and, therefore, $\pi_{*}(\mathscr{R}(M)) \cong \pi_{*} X$ in $\operatorname{Grmod}-\pi_{*} P$. As proj.dim $\pi_{*} X \leq 1$, it follows from Proposition 5.1.1 that

$$
\mathscr{R}(M) \cong X .
$$

Next let us verify fully faithfulness. Let $M, M^{\prime} \in \mathcal{D}\left(\pi_{*} P\right)$ and suppose proj.dim $H_{*} M$ is at most one. Now we check that the morphism $\mathscr{R}:\left[M, M^{\prime}\right] \longrightarrow\left[\mathscr{R}(M), \mathscr{R}\left(M^{\prime}\right)\right]$ is an isomorphism. By Lemma 2.2.2, one can choose $F \in \mathcal{D}\left(\pi_{*} P\right)$ and

$$
F \stackrel{\sigma}{\longrightarrow} M
$$

such that $H_{*} F$ is a projective $\pi_{*} P$-module and $H_{*}(\sigma)$ is an epimorphism. Then, embed $\sigma$ into a distinguished triangle

$$
Y \longrightarrow F \stackrel{\sigma}{\longrightarrow} M \longrightarrow Y[1] .
$$

Since $\sigma$ induces a surjection on $H_{*}$, we have a short exact sequence

$$
0 \longrightarrow H_{*} Y \longrightarrow H_{*} F \longrightarrow H_{*} M \longrightarrow 0 .
$$


As proj.dim $H_{*} M \leq 1$ and $H_{*} F$ is projective, $H_{*} Y$ is projective as well. Therefore, by Lemma 5.1 .7 , the triangle

$$
\mathscr{R}(Y) \longrightarrow \mathscr{R}(F) \stackrel{\mathscr{R}(\sigma)}{\longrightarrow} \mathscr{R}(M) \longrightarrow \mathscr{R}(Y[1]) \cong \Sigma \mathscr{R}(Y)
$$

is antidistinguished. In particular, if we apply $\left[-, \mathscr{R}\left(M^{\prime}\right)\right]$ to this triangle, we get a long exact sequence. Moreover, $\mathscr{R}$ induces a morphism of long exact sequences
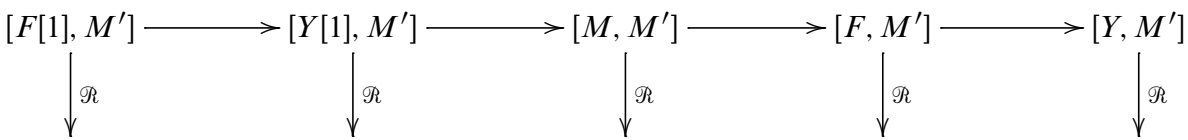

$\left[\mathscr{R}(F[1]), \mathscr{R}\left(M^{\prime}\right)\right] \rightarrow\left[\mathscr{R}(Y[1]), \mathscr{R}\left(M^{\prime}\right)\right] \rightarrow\left[\mathscr{R}(M), \mathscr{R}\left(M^{\prime}\right)\right] \rightarrow\left[\mathscr{R}(F), \mathscr{R}\left(M^{\prime}\right)\right] \rightarrow\left[\mathscr{R}(Y), \mathscr{R}\left(M^{\prime}\right)\right]$.

By Theorem 3.1.4(i), Lemma 2.2.2(i) and the Five Lemma,

$$
\mathscr{R}:\left[M, M^{\prime}\right] \longrightarrow\left[\mathscr{R}(M), \mathscr{R}\left(M^{\prime}\right)\right]
$$

is an isomorphism.

Corollary 5.2.1 Let $\mathcal{M}$ be a stable model category and $P$ a compact generator of $\operatorname{Ho}(\mathcal{M})$. Suppose that the graded ring $\pi_{*} P$ is $N$-sparse and gl.dim $\pi_{*} P=1$. Then the functor

$$
\mathscr{R}: \mathcal{D}\left(\pi_{*} P\right) \longrightarrow \operatorname{Ho}(\mathcal{M})
$$

is an equivalence of categories.

Remark 5.2.2 Lemma 5.1.7 and the construction of the Adams spectral sequence of Proposition 2.2.3 show that $\mathscr{R}$ preserves (up to sign) universal coefficient sequences. More precisely, for any $M \in \mathcal{D}\left(\pi_{*} P\right)$ with proj.dim $H_{*} M \leq 1$ and $M^{\prime} \in \mathcal{D}\left(\pi_{*} P\right)$, the diagram

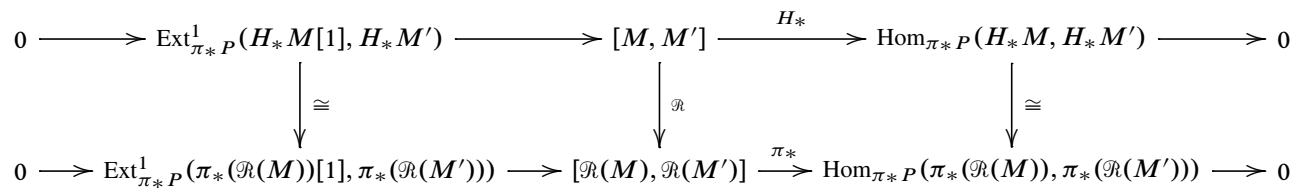

commutes up to sign, where the left and right isomorphisms come from the functor isomorphism

$$
\pi_{*} \circ \mathscr{R} \cong H_{*} .
$$

Note that the equivalence of categories

$$
\mathcal{D}\left(\pi_{*} P\right) \sim \operatorname{Ho}(\mathcal{M})
$$

in Corollary 5.2.1 is a special case of Greenlees [10, 4.3.2]. Moreover, since the functor $\mathscr{R}$ preserves universal coefficient sequences, it is in fact naturally isomorphic 
to the functor constructed in $[10,4.3]$. One should note although $[10,4.3 .2]$ is stated for homotopy categories coming from stable model categories, the proof in [10, 4.3] can be equally applied to general triangulated categories as well. In other words, one has the following generalization of Corollary 5.2.1.

Proposition 5.2.3 Suppose $\mathcal{T}$ is a triangulated category with infinite coproducts, $P \in \mathcal{T}$ a compact generator, and let $\pi_{*}=\operatorname{Hom}_{\mathcal{T}}(P,-)_{*}$. Suppose that the graded ring $\pi_{*} P$ is $N$-sparse and $\mathbf{g l . d i m} \pi_{*} P=1$. Then $\mathcal{T}$ is equivalent to $\mathcal{D}\left(\pi_{*} P\right)$.

Remark 5.2.4 According to $[10,4.3 .1]$ the equivalence $\mathcal{D}\left(\pi_{*} P\right) \sim \operatorname{Ho}(\mathcal{M})$ is even a triangulated equivalence. However, the argument about Toda brackets in [10, 4.3] and hence the proof of the latter claim contains a gap. We do not know whether the equivalences in Proposition 5.2.3 and Corollary 5.2.1 are triangulated or not.

\subsection{Examples}

Example 5.3.1 (Complex $K$-theory) It is well known to specialists that the derived category $\mathcal{D}(K U)$ of the complex $K$-theory spectrum $K U$ is equivalent to the derived category $\mathcal{D}\left(\pi_{*} K U\right)$ of the homotopy ring

$$
\pi_{*} K U \cong \mathbb{Z}\left[u, u^{-1}\right], \quad|u|=2 .
$$

Since $\pi_{*} K U$ is 2 -sparse and gl.dim $\pi_{*} K U=1$, one can think of this equivalence as a consequence of Corollary 5.2.1. Note also that $\Omega^{\infty} K U \simeq \mathbb{Z} \times B U$ is not a product of Eilenberg-Mac Lane spaces (see Corollary A.1.2). Consequently, in view of Corollary 4.2.4, there does not exist a zigzag of Quillen equivalences between the model categories Mod $-K U$ and $\operatorname{Mod}-\mathbb{Z}\left[u, u^{-1}\right]$. In particular,

$$
\mathscr{R}: \mathcal{D}\left(\mathbb{Z}\left[u, u^{-1}\right]\right) \longrightarrow \mathcal{D}(K U)
$$

can not be derived from a zigzag of Quillen equivalences. Note that we do not know whether the equivalence $\mathcal{D}\left(\mathbb{Z}\left[u, u^{-1}\right]\right) \sim \mathcal{D}(K U)$ is triangulated or not.

Example 5.3.2 (Connective Morava $K$-theories) The connective Morava $K$-theory spectrum $k(n), n \geq 1$, is obtained from the truncated Brown-Peterson spectrum $B P\langle n\rangle$ by killing the regular sequence $\left(p, v_{1}, \ldots, v_{n-1}\right)$. In particular, we have an isomorphism of graded rings

$$
\pi_{*} k(n) \cong \mathbb{F}_{p}\left[v_{n}\right], \quad\left|v_{n}\right|=2\left(p^{n}-1\right) .
$$

As gl.dim $\pi_{*} k(n)=1$ and $\pi_{*} k(n)$ is $2\left(p^{n}-1\right)$-sparse, the functor

$$
\mathscr{R}: \mathcal{D}\left(\mathbb{F}_{p}\left[v_{n}\right]\right) \longrightarrow \mathcal{D}(k(n))
$$


is an equivalence of categories by Corollary 5.2.1. Observe that $\Omega^{\infty} k(n)$ is not a product of Eilenberg-Mac Lane spaces (see Proposition A.1.10). Therefore, by Corollary 4.2.4, the model categories $\operatorname{Mod}-k(n)$ and $\operatorname{Mod}-\mathbb{F}_{p}\left[v_{n}\right]$ are not connected by a zigzag of Quillen equivalences. In particular, $\mathscr{R}$ does not come from a zigzag of Quillen equivalences.

\section{The two-dimensional case}

In this section we prove Theorems 3.1.4(iii) and 3.1.5.

\subsection{Proof of Theorem 3.1.4(iii)}

Let $X \in \operatorname{Ho}(\mathcal{M})$ and suppose proj.dim $\pi_{*} X \leq 2$. By Lemma 2.2.2 and Corollary 5.1.2, there are cofibrant objects $F^{(i)}, i \in \mathbb{Z} / N \mathbb{Z}$, and a morphism

$$
\bigvee_{i} F^{(i)} \stackrel{\sigma}{\longrightarrow} X
$$

such that $\pi_{*} F^{(i)}$ are projective, $\pi_{*} F^{(i)} \in \mathcal{B}[i]$ and

$$
\bigoplus_{i} \pi_{*} F^{(i)} \stackrel{\pi_{*} \sigma}{\longrightarrow} \pi_{*} X
$$

is an epimorphism. Embed $\sigma$ into a distinguished triangle

$$
Y \longrightarrow \bigvee_{i} F^{(i)} \stackrel{\sigma}{\longrightarrow} X \longrightarrow \Sigma Y .
$$

Since $\sigma$ induces a surjection on $\pi_{*}$, we have a short exact sequence

$$
0 \longrightarrow \pi_{*} Y \longrightarrow \bigoplus_{i} \pi_{*} F^{(i)} \stackrel{\pi_{*} \sigma}{\longrightarrow} \pi_{*} X \longrightarrow 0 .
$$

As proj.dim $\pi_{*} X \leq 2$ and $\bigoplus_{i} \pi_{*} F^{(i)}$ is projective, one has

$$
\text { proj.dim } \pi_{*} Y \leq 1 \text {. }
$$

Combining this with Corollary 5.1.2, we get a splitting

$$
Y \cong \bigvee Y^{(i)}
$$

in $\operatorname{Ho}(\mathcal{M})$, where $Y^{(i)} \in \mathcal{M}_{\text {cof }}$, and $\pi_{*} \stackrel{i}{Y}^{(i)} \in \mathcal{B}[i], i \in \mathbb{Z} / N \mathbb{Z}$. If we replace $Y$ by $\bigvee_{i} Y^{(i)}$ in (6.1.1), we get a distinguished triangle

$$
\bigvee_{i} Y^{(i)} \longrightarrow \bigvee_{i} F^{(i)} \stackrel{\sigma}{\longrightarrow} X \longrightarrow \Sigma\left(\bigvee_{i} Y^{(i)}\right)
$$


By the Adams spectral sequence of Proposition 2.2.3,

$$
\left[Y^{(i)}, F^{(j)}\right]=0, \quad j \neq i, i+1(\bmod N),
$$

since proj.dim $\pi_{*} Y^{(i)} \leq 1$. Therefore the only possible nontrivial entries of

$$
\bigvee_{i} Y^{(i)} \longrightarrow \bigvee_{i} F^{(i)}
$$

are

$$
Y^{(i)} \stackrel{\alpha_{i}}{\longrightarrow} F^{(i)}, \quad Y^{(i-1) \stackrel{\beta_{i}}{\longrightarrow}} F^{(i)} .
$$

Without loss of generality one may assume that $F^{(i)}$ is bifibrant (ie, cofibrant and fibrant) for any $i \in \mathbb{Z} / N \mathbb{Z}$. Hence, there exist morphisms

$$
l_{i}: Y^{(i)} \longrightarrow F^{(i)}, \quad k_{i}: Y^{(i-1)} \longrightarrow F^{(i)}
$$

in $\mathcal{M}$ whose homotopy classes are $\alpha_{i}$ and $-\beta_{i}$, respectively. Then, we get a diagram

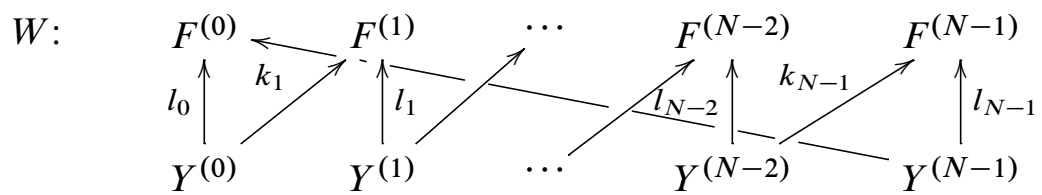

in $\mathcal{M}$, ie, an object of $\mathcal{M}^{\mathcal{C}_{N}}$. We may also assume that $W \in\left(\mathcal{M}^{\mathcal{C}_{N}}\right)_{\text {cof }}$ (otherwise we could replace it cofibrantly). The homotopy colimit of $W$ is isomorphic to the homotopy coequalizer of

$$
\bigvee_{i} Y^{(i)} \underset{k}{\stackrel{l}{\longrightarrow}} \bigvee_{i} F^{(i)}
$$

where $k=\bigvee_{i} k_{i}$ and $l=\bigvee_{i} l_{i}$. Therefore, by Lemma 2.6.4, one has

$$
\text { Hocolim } W \cong X
$$

in $\operatorname{Ho}(\mathcal{M})$. On the other hand, clearly, $W \in \mathcal{K}$. Hence, the construction of $\mathscr{R}$ implies

$$
\mathscr{R}(\mathcal{Q}(W)) \cong X
$$

thus completing the proof.

\subsection{Technical lemmas}

In this subsection we prove two lemmas which will be used in the proof of Theorem 3.1.5. 
Lemma 6.2.1 Let $\mathcal{M}, P, \pi_{*}$ and $N$ be as in Theorem 3.1.5, and $\left(C_{*}, d\right)$ be a $D G \pi_{*} P$-module and $G_{*}$ a $D G \pi_{*} P$-module with zero differential (ie, a graded $\pi_{*} P$-module), and suppose that

$$
f: G_{*} \longrightarrow C_{*}
$$

is a DG morphism which induces a monomorphism on homology. Then the functor

$$
\mathscr{R}: \mathcal{D}\left(\pi_{*} P\right) \longrightarrow \operatorname{Ho}(\mathcal{M})
$$

sends the antidistinguished triangle (see Remark 2.4.8)

$$
G_{*} \stackrel{f}{\longrightarrow} C_{*} \stackrel{\iota}{\longrightarrow} C(f) \stackrel{-\partial}{\longrightarrow} G_{*}[1]
$$

to a distinguished triangle.

Proof The proof goes in the same way as that of Lemma 5.1.4. First we construct a sequence (see Proposition 3.5.1)

$$
X \longrightarrow Y \longrightarrow Z \longrightarrow X^{\#}
$$

in $\mathcal{L}$ (see Notation 3.3.1 and Proposition 3.3.5. Note that $\mathcal{L}=\mathcal{K}$ under the hypotheses of Theorem 3.1.5.) which is mapped to a triangle isomorphic to (6.2.2) after applying $\mathcal{Q}$.

Consider the splitting

$$
G_{*} \cong G^{(0)} \oplus \cdots \oplus G^{(N-1)}
$$

in Grmod $-\pi_{*} P$, where $G^{(i)} \in \mathcal{B}[i], i \in \mathbb{Z} / N \mathbb{Z}$. In view of Proposition 3.2.1(i), there are cofibrant $X_{\zeta_{i}}$ 's with

$$
\pi_{*} X_{\zeta_{i}} \cong G^{(i)}
$$

Clearly, the diagram

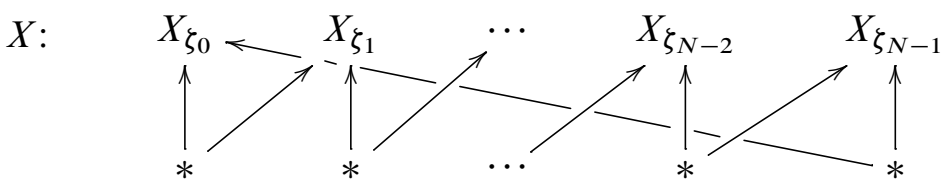

is an object of $\mathcal{L}$ and $\mathcal{Q}(X) \cong\left(G_{*}, 0\right)$. Next, by Corollary 3.3.13, $\mathcal{Q}(\mathcal{L})=\operatorname{Mod}-\pi_{*} P$ since gl.dim $\pi_{*} P$ is less than $N-1$. In particular, one can choose a bifibrant

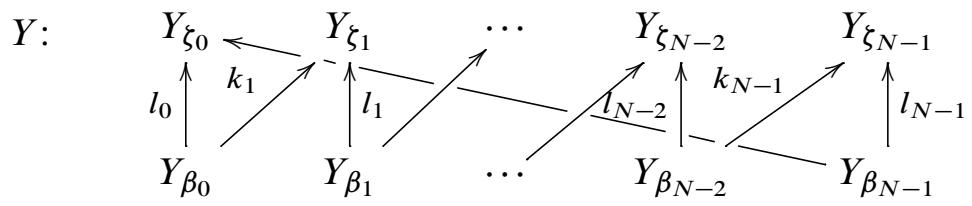


in $\mathcal{L}$ such that

$$
\mathcal{Q}(Y) \cong\left(C_{*}, d\right) .
$$

It then follows from Corollary 3.3.13 that there is a morphism $\psi: X \longrightarrow Y$ in $\mathcal{L}$ so that the diagram

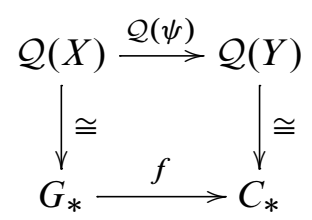

commutes in Mod $-\pi_{*} P$. Further, as $X$ is cofibrant and $Y$ is fibrant, the morphism $\psi$ comes from some morphism in $\mathcal{M}^{\mathcal{C}_{N}}$, denoted by $\psi$ as well. Moreover, using the factorization axiom for the model category $\mathcal{M}^{\mathcal{C}_{N}}$, we may assume that $\psi: X \longrightarrow Y$ is a cofibration in $\mathcal{M}^{\mathcal{C}_{N}}$, ie,

$$
X_{\zeta_{i}} \vee Y_{\beta_{i-1}} \vee Y_{\beta_{i}} \stackrel{\left(\psi_{\zeta_{i}}, k_{i-1}, l_{i}\right)}{\longrightarrow} Y_{\zeta_{i}}
$$

is a cofibration for any $i \in \mathbb{Z} / N \mathbb{Z}$ (see Proposition 2.5.3). Next, we construct an object in $\mathcal{L}$ whose image under $\mathcal{Q}$ is isomorphic to the algebraic mapping cone $C(f)$. Define $Z \in \mathcal{M}^{\mathcal{C}_{N}}$ by

$$
\begin{gathered}
Z_{\zeta_{i}}=C X_{\zeta_{i-1}} \vee Y_{\zeta_{i}}, \quad Z_{\beta_{i}}=X_{\zeta_{i}} \vee Y_{\beta_{i}}, \\
\lambda_{i}: Z_{\beta_{i}}=X_{\zeta_{i}} \vee Y_{\beta_{i}} \stackrel{\left(\psi_{\zeta_{i}}, l_{i}\right)}{\longrightarrow} Y_{\zeta_{i}} \stackrel{\text { incl }}{\longrightarrow} C X_{\zeta_{i-1}} \vee Y_{\zeta_{i}}=Z_{\zeta_{i}}, \\
\kappa_{i}: Z_{\beta_{i-1}}=X_{\zeta_{i-1}} \vee Y_{\beta_{i-1}} \stackrel{\varepsilon_{i-1} \vee k_{i}}{\longrightarrow} C X_{\zeta_{i-1}} \vee Y_{\zeta_{i}}=Z_{\zeta_{i}},
\end{gathered}
$$

where

$$
C(-)=(I, 0) \wedge-,
$$

and $\varepsilon_{i}: X_{\zeta_{i}} \longrightarrow C X_{\zeta_{i}}, i \in \mathbb{Z} / N \mathbb{Z}$, is the canonical map. Thus, the diagram $Z$ looks as follows:

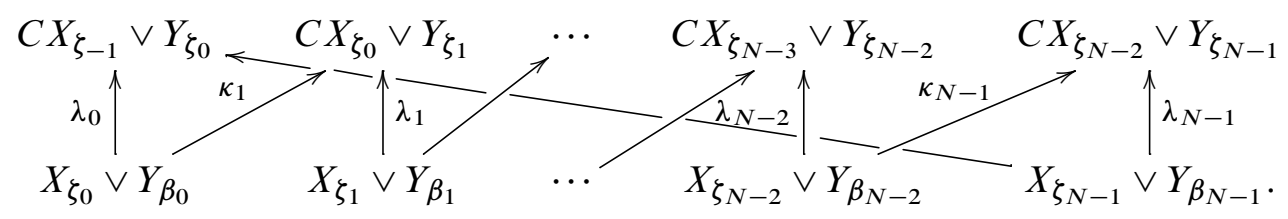

As $X$ and $Y$ are cofibrant and $\psi: X \longrightarrow Y$ is a cofibration, it follows $Z \in\left(\mathcal{M}^{\mathcal{C}_{N}}\right)_{\text {cof }}$. Further,

$$
\pi_{*} \lambda_{i}: \pi_{*} Z_{\beta_{i}} \longrightarrow \pi_{*} Z_{\zeta_{i}}
$$


is a monomorphism since $f$ induces a monomorphism on homology. Besides, obviously, $\pi_{*} Z_{\beta_{i}}$ and $\pi_{*} Z_{\zeta_{i}}$ are objects of $\mathcal{B}[i]$. Consequently, one gets $Z \in \mathcal{L}$. The construction of $\mathcal{Q}$ immediately implies that

$$
\mathcal{Q}(Z) \cong C(f)
$$

in $\operatorname{Mod}-\pi_{*} P$.

The diagram $Z$ comes with two obvious morphisms $Y \longrightarrow Z$ and $Z \longrightarrow X^{\#}$ in $\mathcal{M}^{\mathcal{C}_{N}}$. Hence, we get a sequence

$$
X \stackrel{\psi}{\longrightarrow} Y \longrightarrow Z \longrightarrow X^{\#}
$$

in $\mathcal{L}$. It is immediate from the construction of $\mathcal{Q}$ that the triangle

$$
\mathcal{Q}(X) \stackrel{\mathcal{Q}(\psi)}{\longrightarrow} \mathcal{Q}(Y) \longrightarrow \mathcal{Q}(Z) \longrightarrow \mathcal{Q}\left(X^{\#}\right) \cong \mathcal{Q}(X)[1]
$$

is isomorphic to (6.2.2) (the sign appears by the same reason as in Lemma 5.1.4). On the other hand, applying the functor Hocolim to (6.2.3) ( $X, Y, Z$ are cofibrant) one obtains the elementary distinguished triangle

$$
\operatorname{colim} X \stackrel{\operatorname{colim} \psi}{\longrightarrow} \operatorname{colim} Y \longrightarrow \text { Cone }(\operatorname{colim} \psi) \stackrel{\delta}{\longrightarrow} S^{1} \wedge \operatorname{colim} X .
$$

Now the desired result follows from the construction of $\mathscr{R}$.

Lemma 6.2.4 Let $\mathcal{M}, P, N$ be as in Theorem 3.1.5. Suppose that the triangle

$$
M^{\prime} \stackrel{\iota}{\longrightarrow} F \stackrel{f}{\longrightarrow} M \stackrel{-d}{\longrightarrow} M^{\prime}[1]
$$

is antidistinguished in $\mathcal{D}\left(\pi_{*} P\right)$, and assume that proj.dim $H_{*} M^{\prime} \leq 1$ and $H_{*}(\iota)$ is a monomorphism. Then the triangle

$$
\mathscr{R}\left(M^{\prime}\right) \stackrel{\mathscr{R}(\iota)}{\longrightarrow} \mathscr{R}(F) \stackrel{\mathscr{R}(f)}{\longrightarrow} \mathscr{R}(M) \stackrel{-\mathscr{R}(d)}{\longrightarrow} \mathscr{R}\left(M^{\prime}[1]\right) \cong \Sigma \mathscr{R}\left(M^{\prime}\right)
$$

is distinguished in $\operatorname{Ho}(\mathcal{M})$.

Proof By Proposition 5.1.1, since proj.dim $H_{*} M^{\prime} \leq 1$, one has

$$
M^{\prime} \cong H_{*} M^{\prime}
$$

in $\mathcal{D}\left(\pi_{*} P\right)$, where $H_{*} M^{\prime}$ is regarded as a differential graded $\pi_{*} P$-module with zero differential. Therefore, the triangle (6.2.5) is isomorphic to an antidistinguished triangle

$$
H_{*} M^{\prime} \longrightarrow F \stackrel{f}{\longrightarrow} M \longrightarrow H_{*} M^{\prime}[1] .
$$


Without loss of generality we may assume that $M$ is cofibrant in $\operatorname{Mod}-\pi_{*} P$. Then the last morphism in the latter triangle comes from some morphism $\varphi: M \longrightarrow H_{*} M^{\prime}[1]$ of differential graded modules and, by one of the axioms of triangulated categories, one has a triangle isomorphism

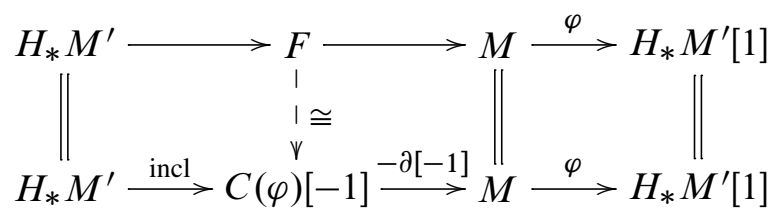

in $\mathcal{D}\left(\pi_{*} P\right)$ (see Remark 2.4.8). On the other hand, the same axiom shows that the lower triangle is isomorphic to

$$
H_{*} M^{\prime} \stackrel{\text { incl }}{\longrightarrow} C(\varphi)[-1] \longrightarrow C(\text { incl }) \stackrel{-\partial}{\longrightarrow} H_{*} M^{\prime}[1] .
$$

Thus the triangle (6.2.5) is isomorphic to an antidistinguished triangle of the form (6.2.2) from Lemma 6.2.1. This completes the proof.

\subsection{Proof of Theorem 3.1.5}

It remains to check that $\mathscr{R}$ is fully faithful when restricted to the full subcategory of at most two dimensional objects. We now verify a more general fact. Namely, we show that for $M \in \mathcal{D}\left(\pi_{*} P\right)$ with proj.dim $H_{*} M \leq 2$ and any $M^{\prime} \in \mathcal{D}\left(\pi_{*} P\right)$, the morphism

$$
\mathscr{R}:\left[M, M^{\prime}\right] \longrightarrow\left[\mathscr{R}(M), \mathscr{R}\left(M^{\prime}\right)\right]
$$

is an isomorphism. Indeed, by Lemma 2.2.2, we may choose $F \in \mathcal{D}\left(\pi_{*} P\right)$ and $\sigma: F \longrightarrow M$ so that $H_{*} F$ is projective and $H_{*}(\sigma)$ an epimorphism. Embed $\sigma$ into a distinguished triangle

$$
Y \longrightarrow F \stackrel{\sigma}{\longrightarrow} M \longrightarrow Y[1] .
$$

As $\sigma$ induces a surjection on $H_{*}$, one has a short exact sequence

$$
0 \longrightarrow H_{*} Y \longrightarrow H_{*} F \stackrel{H_{*}(\sigma)}{\longrightarrow} H_{*} M \longrightarrow 0 .
$$

It then follows that

$$
\text { proj.dim } H_{*} Y \leq 1 \text {, }
$$

since proj.dim $H_{*} M \leq 2$ and $H_{*} F$ is projective. Therefore, by Lemma 6.2.4, the triangle

$$
\mathscr{R}(Y) \longrightarrow \mathscr{R}(F) \stackrel{\mathscr{R}(\sigma)}{\longrightarrow} \mathscr{R}(M) \longrightarrow \mathscr{R}(Y[1]) \cong \Sigma \mathscr{R}(Y)
$$


is antidistinguished. In particular, if we apply $\left[-, \mathscr{R}\left(M^{\prime}\right)\right]$ to this triangle, we get a long exact sequence. Moreover, $\mathscr{R}$ induces a morphism of long exact sequences

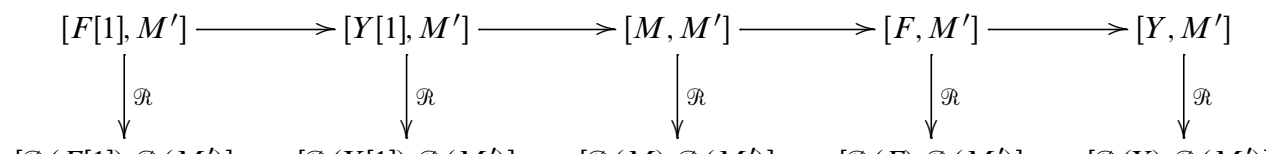

$\left[\Re(F[1]), \mathscr{R}\left(M^{\prime}\right)\right] \longrightarrow\left[\Re(Y[1]), \mathscr{R}\left(M^{\prime}\right)\right] \rightarrow\left[\Re(M), \mathscr{R}\left(M^{\prime}\right)\right] \rightarrow\left[\Re(F), \mathscr{R}\left(M^{\prime}\right)\right] \rightarrow\left[\Re(Y), \mathscr{R}\left(M^{\prime}\right)\right]$.

By Lemma 2.2.2(i), the first and fourth morphisms in the diagram are isomorphisms. Besides, the proof in Subsection 5.2 shows that so are the second and last one. Consequently, by the five lemma,

$$
\mathscr{R}:\left[M, M^{\prime}\right] \longrightarrow\left[\Re(M), \mathscr{R}\left(M^{\prime}\right)\right]
$$

is an isomorphism.

Remark 6.3.1 Let $\mathcal{M}, P$ and $N$ be as in Theorem 3.1.4. In [7, 2.2, Proposition 2] Franke claims that for any distinguished triangle

$$
M \stackrel{f}{\longrightarrow} M^{\prime} \stackrel{g}{\longrightarrow} M^{\prime \prime} \stackrel{\partial}{\longrightarrow} M[1]
$$

in $\mathcal{D}\left(\pi_{*} P\right)$ with $H_{*}(f)$ a monomorphism, the triangle

$$
\mathscr{R}(M) \stackrel{\mathscr{R}(f)}{\longrightarrow} \mathscr{R}\left(M^{\prime}\right) \stackrel{\mathscr{R}(g)}{\longrightarrow} \mathscr{R}\left(M^{\prime \prime}\right) \stackrel{\mathscr{R}(\partial)}{\longrightarrow} \mathscr{R}(M[1]) \cong \Sigma \mathscr{R}(M)
$$

is (anti)distinguished in $\operatorname{Ho}(\mathcal{M})$. If this was so, then one could prove (similarly to the proofs of Theorems 3.1.4(ii) and 3.1.5) that under the hypotheses of Theorem 3.1.4

$$
\mathscr{R}: \mathcal{D}\left(\pi_{*} P\right) \longrightarrow \operatorname{Ho}(\mathcal{M})
$$

is fully faithful. Moreover, using the claim, one can also check that $\mathscr{R}$ is essentially surjective and thus an equivalence of categories. But, the proof of [7, 2.2, Proposition 2] contains a gap. The point is that the arguments given in [7, 2.2], show that the sequence

$$
\mathscr{R}(M) \stackrel{\mathscr{R}(f)}{\longrightarrow} \mathscr{R}\left(M^{\prime}\right) \stackrel{\mathscr{R}(g)}{\longrightarrow} \mathscr{R}\left(M^{\prime \prime}\right)
$$

is a part of some distinguished triangle, but do not say anything about why exactly the morphism

$$
\mathscr{R}\left(M^{\prime \prime}\right) \stackrel{\mathscr{R}(\partial)}{\longrightarrow} \mathscr{R}(M[1]) \cong \Sigma \mathscr{R}(M)
$$

makes this sequence into an (anti)distinguished triangle.

We were unable to fill this gap in the general setting. Although we managed to do this in low dimensional cases and thus obtained the statements of Theorems 3.1.4 and 3.1.5. 
Note that in particular, we do not know whether the functor

$$
\mathscr{R}: \mathcal{D}\left(\pi_{*} P\right) \longrightarrow \operatorname{Ho}(\mathcal{M})
$$

is triangulated in general or not.

\subsection{Examples}

Example 6.4.1 (Real connective $K$-theory localized at an odd prime) Consider $k o_{(p)}$, the real connective $K$-theory spectrum localized at an odd prime $p$. The ring isomorphism

$$
\pi_{*} k o_{(p)} \cong \mathbb{Z}_{(p)}[\omega], \quad|\omega|=4,
$$

implies that gl.dim $\pi_{*} k o_{(p)}=2$ and $\pi_{*} k o_{(p)}$ is 4 -sparse. Thus Corollary 1.1.3 can be applied to $k o_{(p)}$ and therefore we conclude that the functor

$$
\mathscr{R}: \mathcal{D}\left(\mathbb{Z}_{(p)}[\omega]\right) \longrightarrow \mathcal{D}(k o(p))
$$

is an equivalence of categories. Note that $\Omega^{\infty} k o_{(p)} \simeq \mathbb{Z}_{(p)} \times B O_{(p)}$ is not a product of Eilenberg-Mac Lane spaces (see Corollary A.1.3). Hence, in view of Corollary 4.2.4, there does not exist a zigzag of Quillen equivalences between the model categories $\operatorname{Mod}-k o_{(p)}$ and $\operatorname{Mod}-\mathbb{Z}_{(p)}[\omega]$. In particular, the functor

$$
\mathscr{R}: \mathcal{D}\left(\mathbb{Z}_{(p)}[\omega]\right) \longrightarrow \mathcal{D}(k o(p))
$$

can not be derived from a zigzag of Quillen equivalences.

Example 6.4.2 The truncated Brown-Peterson spectrum $B P\langle 1\rangle$ for an odd prime $p$ satisfies the assumptions of Corollary 1.1.3. Indeed, there is a ring isomorphism

$$
\pi_{*} B P\langle 1\rangle \cong \mathbb{Z}_{(p)}\left[v_{1}\right], \quad\left|v_{1}\right|=2(p-1),
$$

and $\operatorname{gld} \operatorname{dim} \mathbb{Z}_{(p)}\left[v_{1}\right]=2$ and $2(p-1) \geq 4$. Thus, the functor

$$
\mathscr{R}: \mathcal{D}\left(\mathbb{Z}_{(p)}\left[v_{1}\right]\right) \longrightarrow \mathcal{D}(B P\langle 1\rangle)
$$

is an equivalence of categories. Next, as mentioned above, $\Omega^{\infty} B P\langle 1\rangle$ is not a product of Eilenberg-Mac Lane spaces (see Corollary A.1.5). Therefore, by Corollary 4.2.4, the model categories Mod $-B P\langle 1\rangle$ and $\operatorname{Mod}-\mathbb{Z}_{(p)}\left[v_{1}\right]$ can not be connected by a zigzag of Quillen equivalences. In particular, $\mathscr{R}: \mathcal{D}\left(\mathbb{Z}_{(p)}\left[v_{1}\right]\right) \longrightarrow \mathcal{D}(B P\langle 1\rangle)$ is not derived from a zigzag of Quillen equivalences.

Example 6.4.3 Another ring spectrum to which Corollary 1.1.3 applies is the JohnsonWilson spectrum $E(2)$ for an odd prime $p$. This follows from the ring isomorphism

$$
\pi_{*} E(2) \cong \mathbb{Z}_{(p)}\left[v_{1}, v_{2}, v_{2}^{-1}\right], \quad\left|v_{1}\right|=2(p-1), \quad\left|v_{2}\right|=2\left(p^{2}-1\right),
$$


since gl.dim $\mathbb{Z}_{(p)}\left[v_{1}, v_{2}, v_{2}^{-1}\right]=2$ and $2(p-1) \geq 4$. Further, as in the two previous examples, $\Omega^{\infty} E(2)$ is not a product of Eilenberg-Mac Lane spaces (see Corollary A.1.8) and therefore, by Corollary 4.2.4, there is no zigzag of Quillen equivalences between the model categories $\operatorname{Mod}-E(2)$ and $\operatorname{Mod}-\mathbb{Z}_{(p)}\left[v_{1}, v_{2}, v_{2}^{-1}\right]$. In particular, the equivalence $\mathscr{R}: \mathcal{D}\left(\mathbb{Z}_{(p)}\left[v_{1}, v_{2}, v_{2}^{-1}\right]\right) \longrightarrow \mathcal{D}(E(2))$ does not come from a zigzag of Quillen equivalences.

Finally, we would like to conclude this section with the following remark.

Remark 6.4.4 As noted above, Corollaries 3.1.7 and 3.1.8 are consequences of Theorems 1.1.1 and 1.1.2, respectively. However, one can also give independent and purely algebraic proofs of Corollaries 3.1.7 and 3.1.8 as well. The main idea is the same as in the proofs of Theorems 3.1.4 and 3.1.5, but in this special case one can use various algebraic constructions which are easier (compared to their topological counterparts) to deal with. Indeed, instead of the cone construction from Definition 2.4.3 we can apply the algebraic mapping cone construction of Remark 2.4.8. Further, the mapping spaces in (3.3.8) can be replaced by the internal Hom-complexes. Next, since any cofibration in Mod $-A$ is a split monomorphism of underlying graded modules, the right vertical arrow in (3.3.8) becomes an epimorphism and thus (3.3.8) leads to a short exact sequence of chain complexes. Clearly, the resulting long exact homology sequence plays the role of the Mayer-Vietoris sequence. Then, the verification of the identity $q \partial=b$ in (3.3.10) can be done by explicit algebraic calculations with homology classes. Finally, the long exact sequence of the homotopy coequalizer (3.3.15) becomes the long exact homology sequence of

$$
0 \longrightarrow \bigoplus_{i} X_{\beta_{i}} \stackrel{l-k}{\longrightarrow} \bigoplus_{i} X_{\zeta_{i}} \longrightarrow \operatorname{colim} X \longrightarrow 0 .
$$

The other points of the proofs of Theorems 3.1.4 and 3.1.5 are more or less formal and can be directly used in the algebraic setting as well.

\section{Appendix A Infinite loop spaces}

In this appendix we show that the infinite loop spaces appearing in Subsections 4.3, 5.3 and 6.4 are not products of Eilenberg-Mac Lane spaces. These facts are well known to experts. However, the author was unable to find proper references for the material. We start with the following.

Lemma A.1.1 Let $X$ be a connected space and $R$ denote one of the rings $\mathbb{Z}$ or $\mathbb{Z}_{(p)}$ (for $p$ a prime). Suppose that $H^{*}(X, R)$ is torsion free and $\pi_{n} X$ has a nontrivial 
$R$-free direct summand for infinitely many $n \in \mathbb{N}$. Then $X$ is not weakly equivalent to a product of Eilenberg-Mac Lane spaces.

Proof Assume that $X$ is weakly equivalent to the product of Eilenberg-Mac Lane spaces

$$
\prod_{n \geq 1} K\left(\pi_{n} X, n\right)
$$

Fix any $m \geq 0$ and choose $s>\max (m, 2)$ so that $\pi_{s} X$ contains a nontrivial $R$-free direct summand. Then the cohomology

$$
H^{m+s}(X, R) \cong H^{m+s}\left(\prod_{n \geq 1} K\left(\pi_{n} X, n\right), R\right)
$$

contains the stable cohomology of Eilenberg-Mac Lane spaces

$$
H^{m+s}(K(R, s), R)=H^{m}(H R, R),
$$

( $s>m$ is the stable range) as a direct summand, for any $m \geq 0$ ( $H R$ denotes the Eilenberg-Mac Lane spectrum associated to $R)$. It is well known that $H^{*}(H R, R)$ has torsion (see eg, Kochman [15, Theorem 3.5]). Thus, we see that $H^{*}(X, R)$ has torsion which is a contradiction.

Corollary A.1.2 The classifying space $B U$ is not weakly equivalent to a product of Eilenberg-Mac Lane spaces. Moreover, for any prime $p$, the localization $B U_{(p)}$ is not weakly equivalent to a product of Eilenberg-Mac Lane spaces.

Proof It is well known (see eg, Switzer [23, 16.11. Corollary]) that

$$
\begin{aligned}
H^{*}(B U, \mathbb{Z}) & \cong \mathbb{Z}\left[c_{1}, c_{2}, \ldots\right], \\
H^{*}\left(B U_{(p)}, \mathbb{Z}_{(p)}\right) & \cong \mathbb{Z}_{(p)}\left[c_{1}, c_{2}, \ldots\right] .
\end{aligned}
$$

Next, $\pi_{2 n} B U \cong \mathbb{Z}$ and $\pi_{2 n} B U_{(p)} \cong \mathbb{Z}_{(p)}$, for $n \geq 1$. Hence, we can apply Lemma A.1.1 and deduce the desired result.

Corollary A.1.3 For any odd prime $p$, the space $B O_{(p)}$ is not weakly equivalent to a product of Eilenberg-Mac Lane spaces.

Proof After $p$-localizing the stable equivalence of spectra

$$
K U \simeq K O \wedge C(\eta),
$$

where $\eta$ is the Hopf map, one obtains a splitting

$$
K U_{(p)} \simeq K O_{(p)} \vee \Sigma^{2} K O_{(p)} .
$$


Passing to associated infinite loop spaces and then to path components, we have $B U_{(p)} \simeq B O_{(p)} \times Y$, for some space Y. Since $H^{*}\left(B U_{(p)}, \mathbb{Z}_{(p)}\right)$ is torsion free, so is $H^{*}\left(B O_{(p)}, \mathbb{Z}_{(p)}\right)$. Further, one has $\pi_{4 n} B O_{(p)} \cong \mathbb{Z}_{(p)}$, for $n \geq 1$. Hence, Lemma A.1.1 applies to $B O_{(p)}$.

Remark A.1.4 The space $B O_{(2)}$ is not a product of Eilenberg-Mac Lane spaces as well. However, we do not need this fact in the paper.

Next, we continue with $\Omega^{\infty} B P\langle 1\rangle$. The Adams splitting

$$
k u_{(p)} \simeq \bigvee_{i=0}^{p-2} \Sigma^{2 i} B P\langle 1\rangle
$$

implies that

$$
B U_{(p)} \simeq \Omega_{0}^{\infty} B P\langle 1\rangle \times Y,
$$

where $\Omega_{0}^{\infty} B P\langle 1\rangle$ is the path component of the unit and $Y$ a certain space. Hence, the cohomology $H^{*}\left(\Omega_{0}^{\infty} B P\langle 1\rangle, \mathbb{Z}_{(p)}\right)$ is torsion free. On the other hand, we have $\pi_{2(p-1) m}\left(\Omega_{0}^{\infty} B P\langle 1\rangle\right) \cong \mathbb{Z}_{(p)}, m \geq 1$. Thus, by Lemma A.1.1, one obtains the following corollary.

Corollary A.1.5 For any prime $p$, the space $\Omega^{\infty} B P\langle 1\rangle$ is not weakly equivalent to a product of Eilenberg-Mac Lane spaces.

Next, we want to show that $\Omega^{\infty} B P\langle n\rangle$ and $\Omega^{\infty} E(n)$ are not products of EilenbergMac Lane spaces for any $n \geq 1$ and $p$ a prime. The strategy is to deduce these facts from Corollary A.1.5 using the following simple lemma.

Lemma A.1.6 Let $f: X \longrightarrow Y$ be a map of simply connected spaces and suppose $\pi_{l} f: \pi_{l} X \longrightarrow \pi_{l} Y$ is a split monomorphism for some fixed $l \geq 2$. If the $l-t h k-$ invariant $k_{l}(X)$ of $X$ is nontrivial, then the $l$-th $k$-invariant $k_{l}(Y)$ of $Y$ is nontrivial.

Proof Recall that by definition, we have that $k_{l}(X) \in H^{l+1}\left(P_{l-1} X, \pi_{l} X\right)$ and $k_{l}(Y) \in H^{l+1}\left(P_{l-1} Y, \pi_{l} Y\right)$, where $P_{l-1}$ is the $(l-1)$-th Postnikov section functor. Next, consider the diagram

$$
\left.H^{l+1}\left(P_{l-1} Y, \pi_{l} Y\right) \stackrel{\left(P_{l-1} f\right)^{*}}{\longrightarrow} H^{l+1}\left(P_{l-1} X, \pi_{l} Y\right) \stackrel{\left(\pi_{l} f\right)_{*}}{\stackrel{1+1}{r}} H^{l-1} X, \pi_{l} X\right) .
$$

By the naturality of $k$-invariants, we get

$$
\left(P_{l-1} f\right)^{*} k_{l}(Y)=\left(\pi_{l} f\right)_{*} k_{l}(X) .
$$

Since $\pi_{l} f$ is a split monomorphism, so is $\left(\pi_{l} f\right)_{*}$ and hence, $\left(\pi_{l} f\right)_{*} k_{l}(X) \neq 0$. This implies that $\left(P_{l-1} f\right)^{*} k_{l}(Y) \neq 0$ and thus, $k_{l}(Y) \neq 0$. 
Corollary A.1.7 For any prime $p$ and $n \geq 1$, the space $\Omega^{\infty} B P\langle n\rangle$ is not weakly equivalent to a product of Eilenberg-Mac Lane spaces.

Proof It follows from Wilson [24, Corollary 5.5] that

$$
\Omega^{\infty} B P\langle n+1\rangle \simeq \Omega^{\infty} B P\langle n\rangle \times Y,
$$

for some space $Y$. An elementary induction argument using Corollary A.1.5 and Lemma A.1.6 completes the proof.

Further, one has the following corollary.

Corollary A.1.8 For any prime $p$ and $n \geq 1$, the space $\Omega^{\infty} E(n)$ is not weakly equivalent to a product of Eilenberg-Mac Lane spaces.

Proof The morphism of spectra $B P\langle n\rangle \longrightarrow E(n)$ gives a map $\Omega_{0}^{\infty} B P\langle n\rangle \longrightarrow \Omega_{0}^{\infty} E(n)$ which induces split monomorphisms an all homotopy groups. Hence, Corollary A.1.7 and Lemma A.1.6 imply the desired result.

Finally, we turn to $\Omega^{\infty} k(n), n \geq 1$. To show that this space is not a product of Eilenberg-Mac Lane spaces, we need an extra argument since all nontrivial homotopy groups of $k(n)$ are torsion. We use the following well-known result.

Proposition A.1.9 (Rudyak [20, IX.7.17]) For a prime $p$ and $n$ greater than or equal to $1, k_{2\left(p^{n}-1\right)}(k(n))=Q_{n}$ is the first nontrivial (stable) $k$-invariant of $k(n)$. (Here, $Q_{n}$ is a specific degree $2 p^{n}-1$ element in the Steenrod algebra $\mathcal{A}_{p}$.)

As a consequence, one obtains the following.

Proposition A.1.10 For any prime $p$ and $n \geq 1$, the space $\Omega^{\infty} k(n)$ is not weakly equivalent to a product of Eilenberg-Mac Lane spaces.

Proof Proposition A.1.9 implies that there is a cofiber sequence of spectra

$$
\Sigma^{2} p^{n}-2 H \mathbb{F}_{p} \longrightarrow P_{2 p^{n}-2} k(n) \longrightarrow H \mathbb{F}_{p} \stackrel{Q_{n}}{\longrightarrow} \Sigma^{2 p^{n}-1} H \mathbb{F}_{p} .
$$

Let $\left\{k(n)_{l}\right\}_{l \geq 0}$ be the $\Omega$-spectrum associated to $k(n)$. If $l>2 p^{n}-1$ (which implies that the cohomology $H^{l+2 p^{n}-1}\left(K\left(\mathbb{F}_{p}, l\right), \mathbb{F}_{p}\right)$ is stable), the latter cofiber sequence induces a fiber sequence of infinite loop spaces

$$
\mathrm{Ev}_{l} P_{2 p^{n}-2} k(n) \longrightarrow K\left(\mathbb{F}_{p}, l\right) \stackrel{Q_{n}}{\longrightarrow} K\left(\mathbb{F}_{p}, l+2 p^{n}-1\right) .
$$


Here $\mathrm{Ev}_{l}$ is the (derived) evaluation functor at level $l$ from the stable homotopy category to the homotopy category of spaces. Obviously, by applying $\mathrm{Ev}_{l}$ to the stable Postnikov tower of $k(n)$, one obtains a Postnikov tower for the space $\operatorname{Ev}_{l} k(n) \simeq k(n)_{l}$. Hence, $Q_{n}$ is the first (unstable) nontrivial $k$-invariant of $k(n)_{l}$ and therefore, $k \overline{(n)_{l}}$ is not weakly equivalent to a product of Eilenberg-Mac Lane spaces for every $l>\overline{2 p^{n}-1}$. Since $k(n)_{l}$ is the $(l-1)$-connected cover of $\mathrm{Ev}_{l} K(n)$, where $K(n)$ is the periodic Morava $K$-theory, it follows that $\operatorname{Ev}_{l} K(n), l>2 p^{n}-1$, is not weakly equivalent to a product of Eilenberg-Mac Lane spaces. On the other hand the periodicity of $K(n)$ yields that

$$
\Omega^{\infty} K(n) \simeq \operatorname{Ev}_{4\left(p^{n}-1\right)} K(n),
$$

and thus, $\Omega^{\infty} K(n)$ is not weakly equivalent to a product of Eilenberg-Mac Lane spaces. Now the obvious weak equivalence $\Omega^{\infty} K(n) \simeq \Omega^{\infty} k(n)$ finishes the proof.

\section{References}

[1] D Benson, H Krause, S Schwede, Realizability of modules over Tate cohomology, Trans. Amer. Math. Soc. 356 (2004) 3621-3668 MR2055748

[2] A K Bousfield, On the homotopy theory of $K$-local spectra at an odd prime, Amer. J. Math. 107 (1985) 895-932 MR796907

[3] K S Brown, Abstract homotopy theory and generalized sheaf cohomology, Trans. Amer. Math. Soc. 186 (1973) 419-458 MR0341469

[4] J D Christensen, Ideals in triangulated categories: phantoms, ghosts and skeleta, Adv. Math. 136 (1998) 284-339 MR1626856

[5] W G Dwyer, J Spaliński, Homotopy theories and model categories, from: "Handbook of algebraic topology", (I M James, editor), North-Holland, Amsterdam (1995) 73-126 MR1361887

[6] E Dyer, J Roitberg, Note on sequences of Mayer-Vietoris type, Proc. Amer. Math. Soc. 80 (1980) 660-662 MR587950

[7] J Franke, Uniqueness theorems for certain triangulated categories possessing an Adams spectral sequence, preprint, K-theory Preprint Archives (1996) Available at http://www . math.uiuc.edu/K-theory/0139/Adams.pdf

[8] S I Gelfand, Y I Manin, Methods of homological algebra, Springer, Berlin (1996) MR1438306

[9] P G Goerss, J F Jardine, Simplicial homotopy theory, Progress in Mathematics 174, Birkhäuser, Basel (1999) MR1711612

[10] J P C Greenlees, Rational $S^{1}$-equivariant stable homotopy theory, Mem. Amer. Math. Soc. 138 (1999) xii+289 MR1483831 
[11] V Hinich, Homological algebra of homotopy algebras, Comm. Algebra 25 (1997) 3291-3323 MR1465117

[12] M Hovey, Model categories, Mathematical Surveys and Monographs 63, American Mathematical Society (1999) MR1650134

[13] M Hovey, B Shipley, J Smith, Symmetric spectra, J. Amer. Math. Soc. 13 (2000) 149-208 MR1695653

[14] D M Kan, A combinatorial definition of homotopy groups, Ann. of Math. 67 (1958) 282-312 MR0111032

[15] S O Kochman, Integral cohomology operations, from: "Current trends in algebraic topology, Part 1”, (R M Kane, S O Kochman, P S Selick, V P Snaith, editors), CMS Conf. Proc. 2, Amer. Math. Soc., Providence, R.I. (1982) 437-478 MR686130

[16] A Lazarev, Towers of MU-algebras and the generalized Hopkins-Miller theorem, Proc. London Math. Soc. 87 (2003) 498-522 MR1990937

[17] S Mac Lane, Categories for the working mathematician, 2nd edition, Graduate Texts in Mathematics 5, Springer, New York (1998) MR1712872

[18] D G Quillen, Homotopical algebra, Lecture Notes in Mathematics 43, Springer, Berlin (1967) MR0223432

[19] C Roitzheim, On the algebraic classification of $K$-local spectra, Homology, Homotopy Appl. 10 (2008) 389-412 MR2426109

[20] Y B Rudyak, On Thom spectra, orientability, and cobordism, Springer Monographs in Mathematics, Springer, Berlin (1998) MR1627486

[21] S Schwede, B E Shipley, Algebras and modules in monoidal model categories, Proc. London Math. Soc. 80 (2000) 491-511 MR1734325

[22] B Shipley, HZ-algebra spectra are differential graded algebras, Amer. J. Math. 129 (2007) 351-379 MR2306038

[23] R M Switzer, Algebraic topology—homotopy and homology, Grundl. Math. Wissen. 212, Springer, New York (1975) MR0385836

[24] W S Wilson, The $\Omega$-spectrum for Brown-Peterson cohomology, II, Amer. J. Math. 97 (1975) 101-123 MR0383390

[25] J J Wolbert, Classifying modules over K-theory spectra, J. Pure Appl. Algebra 124 (1998) 289-323 MR1600317

Mathematisches Institut, Universität Bonn

Endenicher Allee 60, 53115 Bonn, Germany

irpatchk@math.uni-bonn.de

Received: 4 November $2011 \quad$ Revised: 19 July 2012 